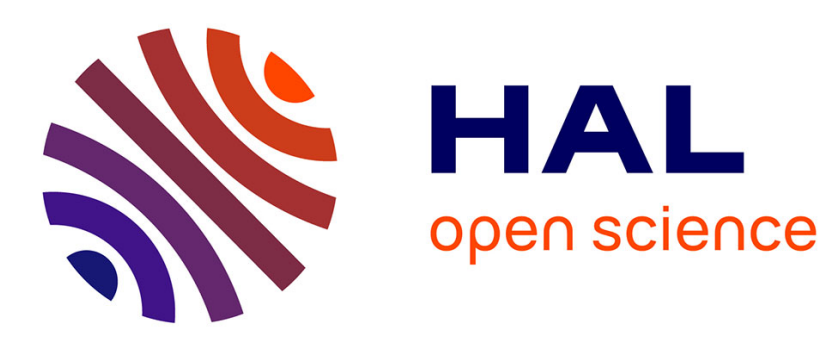

\title{
A sub-Riemannian modular framework for diffeomorphism based analysis of shape ensembles
}

Barbara Gris, Stanley Durrleman, Alain Trouvé

\section{To cite this version:}

Barbara Gris, Stanley Durrleman, Alain Trouvé. A sub-Riemannian modular framework for diffeomorphism based analysis of shape ensembles. SIAM Journal on Imaging Sciences, 2018, 11, pp.802-833. hal-01321142v2

HAL Id: hal-01321142

https://hal.science/hal-01321142v2

Submitted on 15 Nov 2017

HAL is a multi-disciplinary open access archive for the deposit and dissemination of scientific research documents, whether they are published or not. The documents may come from teaching and research institutions in France or abroad, or from public or private research centers.
L'archive ouverte pluridisciplinaire HAL, est destinée au dépôt et à la diffusion de documents scientifiques de niveau recherche, publiés ou non, émanant des établissements d'enseignement et de recherche français ou étrangers, des laboratoires publics ou privés. 


\title{
A SUB-RIEMANNIAN MODULAR FRAMEWORK FOR DIFFEOMORPHISM BASED ANALYSIS OF SHAPE ENSEMBLES
}

\author{
BARBARA GRIS * ${ }^{*}$ STANLEY DURRLEMAN ${ }^{\dagger}$, AND ALAIN TROUVÉ ${ }^{\ddagger}$
}

\begin{abstract}
Deformations, and diffeormophisms in particular, have played a tremendous role in the field of statistical shape analysis, as a proxy to measure and interpret differences between similar objects but with different shapes. Diffeomorphisms usually result from the integration of a flow of regular velocity fields, whose parameters have not enabled so far a full control of the local behaviour of the deformation.

In this work, we propose a new mathematical and computational framework, in which these velocity fields are constrained to be built through a combination of local deformation modules with few degrees of freedom. Deformation modules contribute to the global velocity field, and interact with it during integration so that the local modules are transported by the global diffeomorphic deformation under construction. Such modular diffeomorphisms are used to deform shapes and to provide the shape space with a sub-Riemannian metric.

We then derive a method to estimate a Fréchet mean from a series of observations, and to decompose the variations in shape observed in the training samples into a set of elementary deformation modules encoding distinctive and interpretable aspects of the shape variability. We show how this approach brings new solutions to long lasting problems in the fields of computer vision and medical image analysis. For instance, the easy implementation of priors in the type of deformations offers a direct control to favor one solution over another in situations where multiple solutions may fit the observations equally well. It allows also the joint optimisation of a linear and a non-linear deformation between shapes, the linear transform simply being a particular type of modules.

The proposed approach generalizes previous methods for constructing diffeomorphisms and opens up new perspectives in the field of statistical shape analysis.
\end{abstract}

1. Introduction. Shape data play a very peculiar role within the blooming field of data science. Unlike many other kinds of data, the "right" representation of shape data remains largely an open problem despite a long lasting research effort, for instance in the fields of computer vision and medical image analysis. Another specificity of shape data is their ability to be easily visualized and interpreted. This often demands the derivation of learning methods that yield a statistical estimate also in the form of shapes that may be visualized and interpreted. The definition of generative statistical models is notably difficult due to the inherent structure of shape data, and to the absence of an obvious mathematical framework to define a shape space that accounts for this structure. As a matter of fact, statistical shape analysis still depends on several distinct methodological frameworks [4, 10, 25, 26].

A category of methods builds on the idea to deform one shape onto another to quantify their differences. In these, the deformation is used to position one shape with respect to another in a high-dimensional manifold. This line of works, which follows the pioneering work of Grenander, grounded itself in the seminal vision of D'Arcy Thompson [18, 44], has played an important role in the fields of medical image analysis, computational analysis, or biological shape analysis. In these fields, shape data are derived from 2D or 3D imaging systems, and collections of such data form a set of observations of the same organ, tissue or structure that vary in shape across different individuals. Since such samples are usually observed in full (e.g. without

*CMLA, ENS Cachan, CNRS, Université Paris-Saclay, France AND Inria Paris-Rocquencourt, Sorbonne Universités, UPMC Univ Paris 06 UMR S1127, Inserm U1127, CNRS UMR 7225, ICM, F-75013, Paris, France

†Inria Paris-Rocquencourt, Sorbonne Universités, UPMC Univ Paris 06 UMR S1127, Inserm U1127, CNRS UMR 7225, ICM, F-75013, Paris, France

${ }^{\ddagger}$ CMLA, ENS Cachan, CNRS, Université Paris-Saclay, France 
occlusion) and share a similar topology, it makes sense to try to estimate a diffeomorphic deformation, namely a smooth and invertible space deformation with smooth inverse, which warps one shape onto another. Various mathematical constructions have been derived from this idea [9, 34, 48]. These geometrical constructions have been then included into generative statistical models to estimate an "average shape", usually called "template", and the deformations warping the template to each observation $[2,14,29,30,47]$. The parameters of these deformations are used to give an estimate of the variance of the observations in the tangent-space of the manifold $M$ at the template point. Template and deformations together are referred to as a "statistical atlas", which we will simply call in the following "atlas".

From this point of view, a natural idea is to use, among all the notions of shapes which have been developed [10, 25, 26], the one recently formalized in [4] where shapes are considered as "deformable objects". In this framework, a group of diffeomorphisms $G$ is built by integrating trajectories of vector fields belonging to a fixed Reproducing Kernel Hilbert Space (RKHS) $V$, following the pioneering method of [13] to build diffeomorphic large deformations and the Large Deformations Diffeomorphic Metric Mapping (LDDMM) framework introduced in [45]. A shape space is then defined as a manifold $M$, on which this group $G$ acts. This is the notion of shape that we will use. The inner product of $V$ is used to define a metric, and then a distance, on the shape space $M$. It is shown in [5] that geodesics on $M$ (trajectories whose lengths equal the distance between the starting and the ending point) for this metric are linked to geodesics on $G$ and then to optimal paths of vector fields. It is also shown in [5] that optimal vector fields are always of a certain type which is totally determined by the nature of the shapes of $M$. This is due to a generic reduction principle. Various choices of shape spaces and RKHS [11, 16, 17, 36, 39, 40,41] have led to various frameworks which can be considered as particular cases of this generic framework. For instance, in the case where shapes are landmarks and $V$ is a scalar Gaussian RKHS as in [17], optimal vector fields are necessarily sums of local translations carried by the points of the transported shape. A sparse derivation of this framework introduced in [14] considers geodesic vector fields generated by a small number of landmarks so that they are parametrized in low dimension. Then one can study how these geodesics lying in a low-dimensional space can deform shapes of high dimension: the idea is to find a good dimension to study the variability of a given population. The choice of the authors is to consider geodesic vector fields generated by landmarks, so they are sums of local translations. In [41, 23], authors use the same LDDMM principle on images but they want to obtain geodesic vector fields generated by local generators, so that they can be more easily interpreted. In order to do so they change the data attachment function, which corresponds to using a particular category of shapes defined by the values of the images and their derivatives at certain points of the images. Then they show that the optimal vector fields are generated by higher-order momenta, and then are expressed in function of derivatives of the kernel, allowing locally complex deformations as, for instance, locally affine ones. This allows to obtain vector fields locally more adapted to the shape data, and leads to a representation of deformations in a more compact and interpretable way.

However these models (as they derive from the application of a reduction principle) do not allow to develop a framework where the nature of the vector fields is set a priori, and is independent of the attachment function and the representation of the shape. It is useful for interpretation purpose, and it can be necessary in some cases, to impose beforehand a certain pattern of deformation. For instance, this may be 
relevant to encode prior biological knowledge in the statistical analysis, or to give a way to favour a particular kind of solution if the optimization problem has multiple solutions.

This is one of the reasons why parametric approaches, in which deformations are structured beforehand, were developed. In these frameworks, vector fields at each time are constrained to be a sum of a few interpretable vector fields, parametrized in small dimension by control variables, so that the deformation pattern is controlled and can be interpreted from these controls' values. Typical types of such local patterns may include local scaling, torque, or fanning pattern for instance. The idea is here to reduce the set of considered diffeomorphisms in order to understand them better and to improve their specificity to a given problem. In the Grow Random Iterated Diffeomorphisms (GRID) model [20, 35, 43], deformation patterns are built based on active points, called seeds, whose placements are modelled as a Poisson process and which generate particular local deformations. Locations of seeds are estimated at each time so that they maximize a likelihood: they are not transported by the diffeomorphism during time and then do not follow the evolution of the shape data. In a poly-affine framework introduced in $[8,7]$, authors build trajectories of vector fields $\sum_{i} w_{i} v_{i}$ where $w_{i}$ are localizing functions defined by anchor points, and $v_{i}$ are vector fields such that their flows define an affine transformation. This poly-affine framework is particularly adapted to certain situations, for instance in the registration of bones motion [38] or for cardiac motions [31, 37]. However, the anchor points defining weights $w_{i}$ remain fixed during the integration of the flow. This can prevent very large deformations as the part of the shape that is supposed to be displaced by a certain affine transformation can leave the corresponding area.

A general modelling issue is to know how to update these vector fields and the geometrical parameters defining them during the integration in order to generate large diffeomorphic deformations. A way to tackle this problem is then not only to combine local deformation generators into a single vector field, but also to transport the local generators with the deformation that results from the integration of the velocity fields. Such a framework is introduced in [50] where local constraints depending on the shape allow a particular control on the structure of vector fields. Besides, this structure evolves along the resulting flow and induces a sub-Riemannian structure on the shape space. This framework allows to define local deformation generators and their evolution during the integration of the deformation. However, for numerical reasons, vector fields are constrained to be sums of local translations and then cannot be generically adapted to a given problem.

A general difficulty in all the frameworks presented above is that there is no possibility to define a cost associated to the generated vector fields which would be coherent with the introduced priors: this cost is always given by the norm of the vector field in a fixed space of vector fields. Then if two choices of generators allow to build the same vector fields while one choice is more natural than the other, their costs will be equal: there is no way to favour one type of decomposition over the other.

From what precedes, a natural deformation model should allow the definition of a metric on shape spaces similarly to the frameworks of the LDDMM [33, 49, 51] and diffeons [50]. A second natural requirement would be for the deformations to be locally more complex than local translations similarly to $[41,8,35]$ and in addition to allow the geometrical supports of the deformation (for instance control points) to be different from the shape data as in the frameworks presented in [15, 8, 35]. A last interesting quality would be to define the possible deformation patterns as priors as 
in the models of $[8,35,50]$. The deformation model presented in this article aims at combining all these features.

The main contribution of this work is the definition of a sound and coherent mathematical framework allowing the construction of "modular" diffeomorphisms, which are defined by a set of infinitesimal deformation generators called deformation modules. These modules combine in order to generate velocity fields embedded in a common Hilbert space $V$. Deformations resulting from the integration of trajectories of such velocity fields are used to update the parameters of the modules. This construction forms a dynamical system with a feedback loop, in which modules interact in a dynamical way. Shapes can be deformed by these modular diffeomorphisms, and a concept of cost associated to each module equips the shape space with a sub-Riemannian metric. This structure in general is not Riemannian because of the constraints on the velocity field. Note that even though generated vector fields belong to a common Hilbert space $V$, the cost associated to each module can be chosen almost freely and is not directly linked to the norm of $V$. In particular, we show that in order to ensure the existence of geodesics, one only has to check that an inequality called Uniform Embedding Condition (see Definition 3) is satisfied. We define modules and costs, in a way such that elementary modules may be combined into more complex compound modules, thus allowing the definition of a tree structure for modules. The deformation is determined by the root module, which may be decomposed in a hierarchy of sub-modules. Modular diffeomorphisms allow an explicit control of the local structure of the deformation, in the sense that one may choose beforehand the type and scale of the local deformation in each region of the ambient space. Using a particular deformation module to study a population of shapes corresponds to having a particular prior on this population. The parameters of the vector fields generated by a deformation module have an intuitive meaning, allowing an interpretation of optimal vector fields. They depict the dynamical modular structure of the deformation. An interesting feature of this construction is that it encompasses previous models of diffeomorphic deformations, such as those in [14, 34]. Our paper extends the work of [22] where the notion of deformation modules had been defined in a slightly different way (the notion of Uniform Embedding Condition was directly integrated in the definition of deformation module) and only preliminary theoretical and numerical results were presented.

In Section 2, we present the geometrical construction of the modular diffeomorphisms and in the following Section 3 we explain how this framework can be used to build a sub-Riemannian distance on a shape space. In Section 4, we include such a deformation model in a method to build an atlas from a series of shape data. In Section 5 we illustrate our model on simple but yet challenging problems (a summary table of the used deformation modules is given in Appendix B). The notation is summarized in Appendix A.

\section{Modular large deformation.}

2.1. Deformation Module. We first recall the mathematical framework that defines deformations as the result of the integration of time-varying velocity fields. Let $d$ and $\ell$ be non-zero integers, we define $C_{0}^{\ell}\left(\mathbb{R}^{d}\right)$ as the space of vector fields of class $C^{\ell}$ on $\mathbb{R}^{d}$ whose derivatives of order less than or equal to $\ell$ converge to zero at infinity, and we equip it with the norm $|v|_{\ell}=\sup \left\{\left|\frac{\partial^{\ell_{1}+\cdots+\ell_{d}} v(x)}{\partial x_{1}^{\ell_{1} \cdots x_{d}^{\ell_{d}}} \mid}\right| x \in \mathbb{R}^{d},\left(\ell_{1}, \cdots, \ell_{d}\right) \in\right.$ $\left.\mathbb{N}^{d}, \ell_{1}+\cdot+\ell_{d} \leq \ell\right\}$ making it a Banach space. We let $\operatorname{Diff}_{0}^{\ell}\left(\mathbb{R}^{d}\right)$ be the space of $C^{\ell}$-diffeomorphisms of $\mathbb{R}^{d}$ that converge to identity at infinity, that is an open set 
of the affine Banach space $I d+C_{0}^{\ell}\left(\mathbb{R}^{d}\right)$ and as such equipped with a natural smooth differential structure. We will consider particular trajectories of Diff ${ }_{0}^{\ell}\left(\mathbb{R}^{d}\right)$ defined as flows of specific trajectories of $C_{0}^{\ell}\left(\mathbb{R}^{d}\right)$.

Proposition 1. [6] Let $v$ be an element of $L^{1}\left([0,1], C_{0}^{\ell}\left(\mathbb{R}^{d}\right)\right)$, i.e. a time-dependent vector field such that $t \in[0,1] \mapsto|v(t)|_{\ell}$ is integrable. Then there exists a unique absolutely continuous solution $\varphi^{v}$, called the flow of $v$, to the system

$$
\left\{\begin{aligned}
\dot{\varphi}^{v}(t) & =v(t) \circ \varphi^{v}(t) \\
\varphi(0) & =I d,
\end{aligned}\right.
$$

where $\varphi^{v}(t) \in \operatorname{Diff}_{0}^{\ell}\left(\mathbb{R}^{d}\right)$ for any $t \in[0,1]$.

This proposition allows us to consider flows of time-dependent vector fields, where "time" $t$ refers here to a variable of integration. As we are interested in how these flows can deform a certain shape, we need to make precise the notion of shape, and to specify the way a diffeomorphism of $\operatorname{Diff}_{0}^{\ell}\left(\mathbb{R}^{d}\right)$ can act on it. Several notions of shapes have been formalized $[6,10,25,26]$ but the most adapted to our framework is the one defined by $\mathrm{S}$. Arguillère in [6], which we recall here.

Definition 1. Let $\mathcal{O}$ be a manifold of finite dimension and $k \in \mathbb{N}^{*}$. Assume that the group $\operatorname{Diff}_{0}^{\ell}\left(\mathbb{R}^{d}\right)$ acts continuously on $\mathcal{O}$, according to the action

$$
\begin{aligned}
\operatorname{Diff}_{0}^{\ell}\left(\mathbb{R}^{d}\right) \times \mathcal{O} & \rightarrow \mathcal{O} \\
(\phi, o) & \mapsto \phi \cdot o .
\end{aligned}
$$

We say that $\mathcal{O}$ is a $C^{k}$-shape space of order $\ell$ on $\mathbb{R}^{d}$ if the following conditions are satisfied:

1. For each $o \in \mathcal{O}, \phi \in \operatorname{Diff}_{0}^{\ell}\left(\mathbb{R}^{d}\right) \mapsto \phi \cdot o$ is Lipschitz with respect to the norm $|\cdot|_{\ell}$ and is differentiable at $I d_{\mathbb{R}^{d}}$. This differential is denoted by $\xi_{o}$ and is called the infinitesimal action of $C_{0}^{\ell}\left(\mathbb{R}^{d}\right)$.

2. The mapping $\xi:(o, v) \in \mathcal{O} \times C_{0}^{\ell}\left(\mathbb{R}^{d}\right) \mapsto \xi_{o} v$ is continuous and its restriction to $\mathcal{O} \times C_{0}^{\ell+k}\left(\mathbb{R}^{d}\right)$ is of class $C^{k}$.

An element o of $\mathcal{O}$ is called a shape, and $\mathbb{R}^{d}$ will be referred to as the ambient space.

The simplest example of shape space is the one of landmarks, where shapes are given as a collection of a fixed number of points: $\mathcal{O}=\left\{o=\left(x_{1}, \cdots, x_{n}\right) \in\left(\mathbb{R}^{d}\right)^{n} \mid\right.$ $x_{i} \neq x_{j}$ if $\left.i \neq j\right\}$. The action of $\operatorname{Diff}_{0}^{\ell}\left(\mathbb{R}^{d}\right)$ is given by $\phi \cdot o=\left(\phi\left(x_{1}\right), \cdots, \phi\left(x_{n}\right)\right)$, namely the deformed shape, and the infinitesimal action for $v \in C_{0}^{\ell}\left(\mathbb{R}^{d}\right)$ is $\xi_{o}(v)=$ $\left(v\left(x_{1}\right), \cdots, v\left(x_{n}\right)\right)$ for $o=\left(x_{1}, \cdots, x_{n}\right) \in \mathcal{O}$, namely the velocity of the trajectories of shape points. These actions make $\mathcal{O}$ a shape space of order $\ell$ for any $\ell \geq 1$.

Another example is a set of $N$ Delta Dirac 1-currents encoding tangents of a $2 \mathrm{D}$ or 3D curve [16]. Shapes in this shape space are represented by base points $c_{k}$ and tangent vectors $\tau_{k}$. The action of $\operatorname{Diff}_{0}^{\ell}\left(\mathbb{R}^{d}\right)$ (where $d=2$ or 3 ) is given by $\phi .\left(\left(c_{1}, \tau_{1}\right), \ldots,\left(c_{N}, \tau_{N}\right)\right)=\left(\left(\phi\left(c_{1}\right), d_{c_{1}} \phi\left(\tau_{1}\right)\right), \ldots,\left(\phi\left(c_{N}\right), d_{c_{N}} \phi\left(\tau_{N}\right)\right)\right)$, and the infinitesimal action as $\left(\left(v\left(c_{1}\right), d v_{c_{1}}\left(\tau_{1}\right)\right), \ldots,\left(v\left(c_{N}\right), d v_{c_{1}}\left(\tau_{N}\right)\right)\right)$.

Remark 1. If $\mathcal{O}_{1}$ and $\mathcal{O}_{2}$ are two shape spaces of order $\ell$ on $\mathbb{R}^{d}$, then $\mathcal{O}=\mathcal{O}_{1} \times \mathcal{O}_{2}$ is also a shape space of order $\ell$ on $\mathbb{R}^{d}$.

The following proposition was proved in [6], and shows that a shape transported by a flow of diffeomorphisms (see Proposition 1) satisfies a temporal differential equation. Proposition 2. For every $o \in \mathcal{O}$, the mapping $\phi \mapsto \phi \cdot o$ is of class $C^{1}$. Moreover, if $v \in L^{1}\left([0,1], C_{0}^{\ell}\left(\mathbb{R}^{d}\right)\right)$, for $a \in \mathcal{O}$, the curve $o: t \in[0,1] \mapsto o(t)=\varphi^{v}(t) \cdot a$ is absolutely continuous and satisfies for almost every $t \in[0,1], \dot{o}=\xi_{o}(v)$. 
2.1.1. Definition and first examples. We will now define the core concept of our approach: the notion of deformation module. Intuitively, a deformation module is a structure that embeds a vector field generation mechanism. This mechanism depends on some geometrical descriptors, which specify the local structure of the induced vector fields (think about the positioning of some actuators), and a finite numbers of control parameters commanding the actuators. For a given positioning, a cost is specified for any possible value of the control parameters, so that optimal policies can be defined. For each resulting vector field, a feedback mechanism is defined to update the positioning of the geometrical descriptors. A natural requirement is for this feedback mechanism to be coherent with the action of vector fields (and then diffeomorphisms) on the ambient space. This means that the geometrical descriptors should be transported by a trajectory of vector fields in a natural way compared to the global deformation of the ambient space. This desirable consistency property leads to consider the geometrical descriptors as a shape on which diffeomorphisms act. Hence, following [50], the positioning of the geometrical descriptors will be represented as a shape in a shape space.

In the following we set $d \in \mathbb{N}^{*}$ as the dimension of the ambient space.

Definition 2. Let $k, \ell \in \mathbb{N}^{*}$. We say that $M=(\mathcal{O}, H, \zeta, \xi, c)$ is a $C^{k}$-deformation module of order $\ell$ with geometrical descriptors in $\mathcal{O}$, controls in $H$, infinitesimal action $\xi$, field generator $\zeta$ and cost $c$, if

- $\mathcal{O}$ is a $C^{k}$-shape space of $\mathbb{R}^{d}$ of order $\ell$ with infinitesimal action $\xi: C_{0}^{\ell}\left(\mathbb{R}^{d}\right) \times$ $\mathcal{O} \longrightarrow T \mathcal{O}$

- $H$ is a finite-dimensional Euclidean space,

- $\zeta:(o, h) \in \mathcal{O} \times H \rightarrow\left(o, \zeta_{o}(h)\right) \in \mathcal{O} \times C_{0}^{\ell}\left(\mathbb{R}^{d}\right)$ is continuous, with $h \mapsto \zeta_{o}(h)$ linear and $o \mapsto \zeta_{o}$ of class $C^{k}$,

- $c:(o, h) \in \mathcal{O} \times H \rightarrow c_{o}(h) \in \mathbb{R}^{+}$is a continuous mapping such that $o \mapsto c_{o}$ is smooth and for all $o \in \mathcal{O}, h \mapsto c_{o}(h)$ is a positive quadratic form on $H$, thus defining a smooth metric on $\mathcal{O} \times H$.

A deformation module is defined by the way it can generate a vector field, which is given by the field generator $\zeta$, and by the feedback action on vector fields, which is given by the infinitesimal action $\xi$. A schematic view of the construction of a module can be seen in figure 2.1 .

Remark 2. The geometrical descriptors of a deformation module are considered as a "shape" in the shape space $\mathcal{O}$ in the sense that they satisfy Definition 1 . In the following examples, these "shapes" are the centers of the scaling, of the rotation or the base points of translation vectors. These "shapes" may or may not coincide with points from the input shape data set. For example, as emphasized in the introduction, it is sensible not to locate the center of a scaling on the boundaries of an object but to allow it to be more central. We will see in the following that the input shape data may be considered as geometrical descriptors of a particular module, called a silent module, which is deformed according to its infinitesimal action, but which does not contribute to the global velocity field.

We will now introduce two examples of simple deformation modules (a richer presentation of different deformation modules will be given in Section 2.3). In these first examples we present the construction of deformation modules generating vector fields that can be found in the literature and then show how previous frameworks can be incorporated in our modular model. 


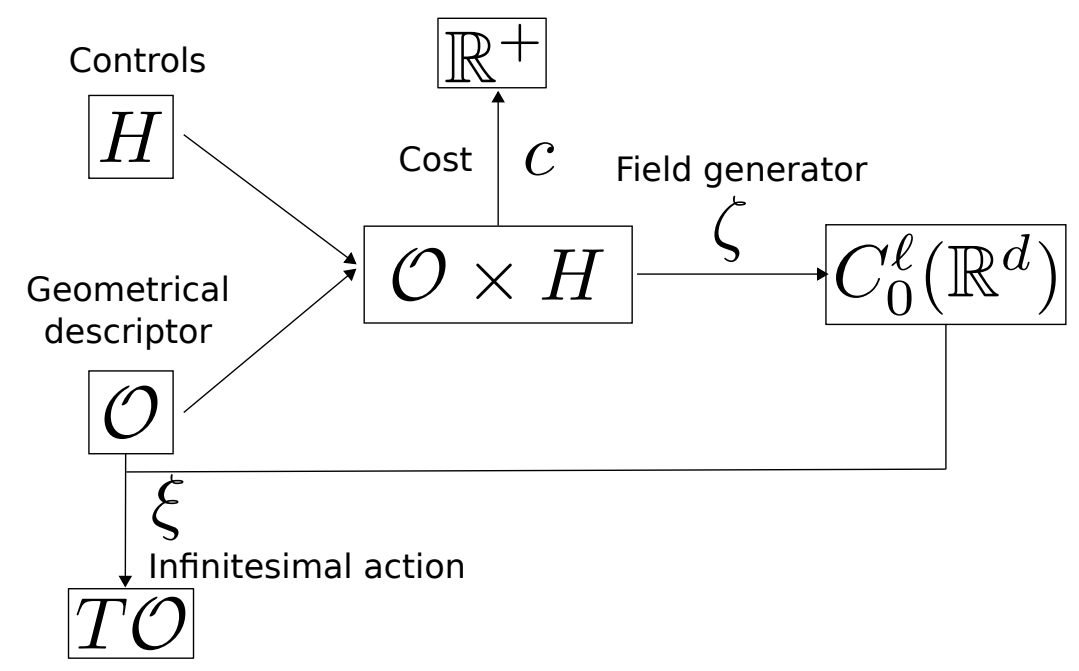

FIG. 2.1. Schematic view of a deformation module.

First example: sum of local translations. This first example explains how the construction of [14] can be seen as a $C^{k}$-deformation module of order $\ell$ for any $k, \ell \in$ $\mathbb{N}^{*}$. We want to build a deformation module $M$ generating vector fields that are a sum of $D$ local translations acting at scale $\sigma$ in the ambient space $\mathbb{R}^{d}\left(d \in \mathbb{N}^{*}\right)$. We let $V_{\sigma}$ be the scalar Gaussian Reproducing Kernel Hilbert Space (RKHS) of scale $\sigma$ (its kernel will be denoted by $K_{\sigma}:(x, y) \in \mathbb{R}^{d} \times \mathbb{R}^{d} \mapsto \exp \left(-|x-y|^{2} / 2 \sigma^{2}\right)$ ), and $\mathcal{O} \doteq\left(\mathbb{R}^{d}\right)^{D}$ the shape space of $D$ landmarks with infinitesimal action $\xi_{o}: v \in$ $C_{0}^{\ell}\left(\mathbb{R}^{d}\right) \mapsto\left(v\left(z_{i}\right)\right)_{i}$, where $o=\left(z_{i}\right) \in \mathcal{O}$ (application of the vector field at each point), and $H \doteq\left(\mathbb{R}^{d}\right)^{D}$ (families of $D$ vectors). For $o=\left(z_{i}\right) \in \mathcal{O}$, we define $\zeta_{o}: h=$ $\left(\alpha_{i}\right) \in H \mapsto \sum_{i=1}^{D} K_{\sigma}\left(z_{i}, \cdot\right) \alpha_{i}$ and $c_{o}: h=\left(\alpha_{i}\right) \in H \mapsto\left|\sum_{i} K_{\sigma}\left(z_{i}, \cdot\right) \alpha_{i}\right|_{V_{\sigma}}^{2}$. It is straightforward to show that $M=(\mathcal{O}, H, \zeta, \xi, c)$ defines a deformation module of order $\ell$.

Second example: local scaling or rotations. In the purpose of interpreting the estimated deformations, a natural step is to build vector fields that are a combination of local affine deformations $[7,38,52]$. We present here the example of deformation modules generating such locally affine deformations. Let $\ell, k \in \mathbb{N}^{*}$. We first detail the construction of a $C^{k}$-deformation module of order $\ell$ which generates vector fields that are local scalings of fixed scale $\sigma \in \mathbb{R}^{+}$in the ambient space $\mathbb{R}^{2}$. A local scaling is parametrized by its center $o \in \mathbb{R}^{2}$ and its scale factor $h \in \mathbb{R}$. The point $o$ plays the role of the geometrical descriptor, and the factor $h$ the control parameter. From $o$ (and the fixed parameter $\sigma$ ), we build 3 points: $z_{1}(o, \sigma)=o+\frac{\sigma}{3}\left(\frac{1}{2} \sqrt{3},-\frac{1}{2}\right), z_{2}(o, \sigma)=$ $o+\frac{\sigma}{3}\left(-\frac{1}{2} \sqrt{3},-\frac{1}{2}\right), z_{3}(o, \sigma)=o+\frac{\sigma}{3}(0,1)$; and 3 unit vectors: $d_{1}(o, \sigma)=\left(\frac{1}{2} \sqrt{3},-\frac{1}{2}\right)$, $d_{2}(o, \sigma)=\left(-\frac{1}{2} \sqrt{3},-\frac{1}{2}\right), d_{3}(o, \sigma)=(0,1)$. They are plotted in Figure 2.2. The idea is to build the vector field generated by the geometrical descriptor $o$ and the control $h$ as an interpolation of the values at these points $z_{j}(o)$ via vectors $d_{j}: \zeta_{o}(h) \doteq$ $h \sum_{j=1}^{3} K_{\sigma}\left(z_{j}(o), \cdot\right) d_{j}$, where $K_{\sigma}$ is the kernel of the scalar Gaussian RKHS of scale $\sigma$. We emphasize here that points $z_{j}(o)$ and vectors $d_{j}$ are intermediate tools used to build the vector field, but that the latter is only parametrized by $o$ and $h$. We then define the deformation module $M$ by the following spaces : $\mathcal{O} \doteq \mathbb{R}^{2}, H=\mathbb{R}$ 


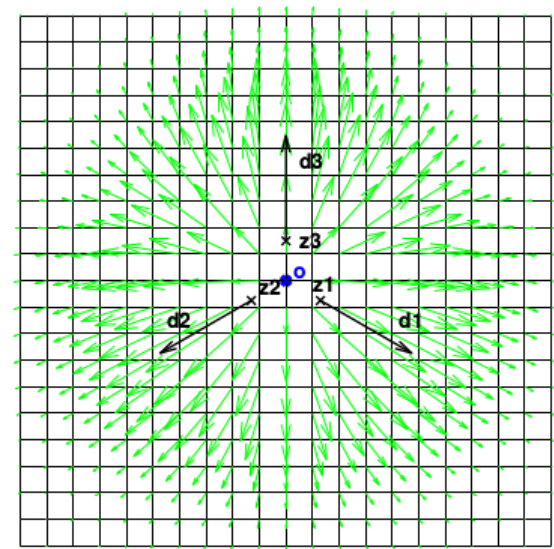

Fig. 2.2. Local scaling. Geometrical descriptor o (in blue) and intermediate tools (in black).scriptor o (in blue) and intermediate tools (in Plot of the resulting vector field in green.

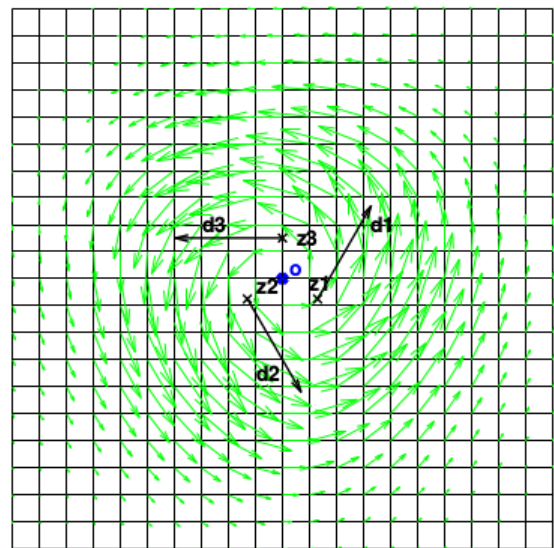

FIG. 2.3. Local rotation. Geometrical deblack). Plot of the resulting vector field in green.

and the following mappings : for $o \in \mathcal{O}, \zeta_{o}: h \in H \mapsto \zeta_{o}(h)$ as given above, $\xi_{o}: v \in C_{0}^{\ell}\left(\mathbb{R}^{2}\right) \mapsto v(o)$, the velocity field at the scaling center, and $c_{o}: h \in H \mapsto$ $\left|\zeta_{o}(h)\right|_{V_{\sigma}}^{2}=h^{2} \sum_{j, j^{\prime}} K_{\sigma}\left(z_{j}, z_{j^{\prime}}\right) d_{j}^{T} d_{j^{\prime}}$, the squared norm of the generated velocity field in the RKHS $V_{\sigma}$. Then $M=(\mathcal{O}, H, \zeta, \xi, c)$ defines a $C^{k}$-deformation module of order $\ell$. Indeed the space $\mathcal{O}$ is a $C^{k}$-shape space (of one landmark) of order $\ell$ and $\zeta: o \in \mathcal{O} \mapsto \zeta_{o} \in L\left(H, C_{0}^{\ell}\left(\mathbb{R}^{2}\right)\right)$ is locally Lipschitz because distances between $z_{i}(o)$ and $z_{i}\left(o^{\prime}\right)(1 \leq i \leq 3)$ are the same as the distance between $o$ and $o^{\prime}$. For the same reason, $\zeta$ is globally continuous. Other properties can be easily verified. This construction can be generalized to any other affine deformation by defining the same points $z_{j}$ but changing the rule according to which vectors $d_{j}$ are built. For example, in Figure 2.3, the generated vector field is a local rotation and is built with vectors $d_{1}(o, \sigma)=\left(\frac{1}{2}, \frac{1}{2} \sqrt{3}\right), d_{2}(o, \sigma)=\left(\frac{1}{2},-\frac{1}{2} \sqrt{3}\right), d_{3}(o, \sigma)=(-1,0)$.

2.1.2. Defining a consistent cost. A key point in the design of a consistent approach for deformation modules is the possibility to deduce existence of optimal deformations between shapes as solutions of the optimal control problem associated with the choice of a cost for the control parameters. This geometrical construction will be detailed in Section 3. This requirement imposes constraints on the choice of costs. A first possibility, following the idea of the LDDMM [4, 33, 49, 51], could be to choose the cost as the squared-norm of the generated vector field. Here, we intend to build a framework where this cost can be chosen more freely but it seems nevertheless important for it to be related with some metric of the induced vector field. Indeed then, controlling the cost of a path of vector fields generated by the deformation module implies controlling the $L^{2}$ norm of this path (and ensures for instance the integrability of this trajectory, see Proposition 4). This is the spirit of the following embedding condition:

Definition 3. Let $M=(\mathcal{O}, H, \zeta, \xi, c)$ be a $C^{k}$-deformation module of order $\ell$. We say that $M$ satisfies the Uniform Embedding Condition (UEC) if there exists a Hilbert space of vector fields $V$ continuously embedded in $C_{0}^{\ell+k}\left(\mathbb{R}^{d}\right)$ and a constant 


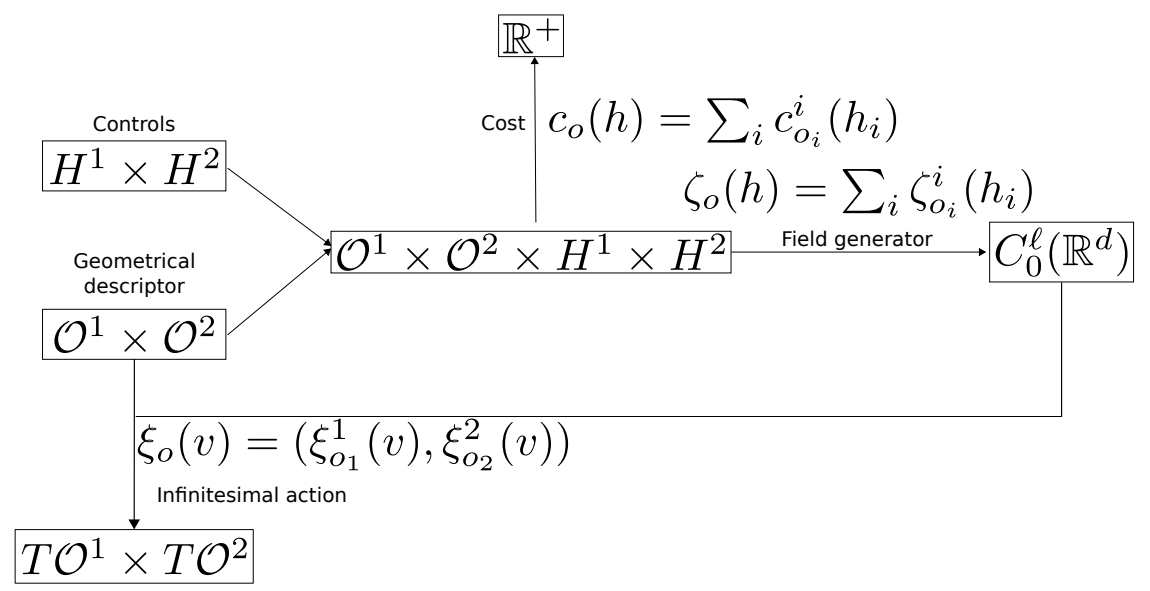

Fig. 2.4. Schematic view of a combination of two deformation modules.

$C>0$ such that for all $o \in \mathcal{O}$ and for all $h \in H, \zeta_{o}(h) \in V$ and

$$
\left|\zeta_{o}(h)\right|_{V}^{2} \leq C c_{o}(h) \text {. }
$$

Remark 3. The previously presented examples of deformation modules satisfy this $U E C$. Indeed in these two examples the infinitesimal action $\xi$ takes values in the Hilbert space $V_{\sigma}$ which is continuously embedded in $C_{0}^{\ell+k}\left(\mathbb{R}^{d}\right)$ (see [16]), and the cost is defined by the squared-norm of the generated vector field in $V_{\sigma}$.

2.1.3. Combination. We have presented in the previous section examples of simple, base deformation modules generating simple vectors fields. We now want to build multi-modular vector fields by summing vector fields generated by different deformation modules. In order to do so, we define the combination of modules as follows (a schematic view of this combination can be seen in Figure 2.4).

Definition 4. Let $M^{l}=\left(\mathcal{O}^{l}, H^{l}, \zeta^{l}, \xi^{l}, c^{l}\right), l=1 \cdots L$, be $L C^{k}$-deformation modules of order $\ell$. We define the compound module of modules $M^{l}$ by $\mathcal{C}\left(M^{l}, l=1 \cdots L\right)=$ $(\mathcal{O}, H, \zeta, \xi, c)$ where $\mathcal{O} \doteq \prod_{l} \mathcal{O}^{l}, H \doteq \prod_{l} H^{l}$ and for $o=\left(o^{l}\right)_{l} \in \mathcal{O}, \zeta_{o}: h=\left(h^{l}\right) \in$ $H \mapsto \sum_{l} \zeta_{o^{l}}^{l}\left(h^{l}\right), \xi_{o}: v \in C_{0}^{\ell}\left(\mathbb{R}^{d}\right) \mapsto\left(\xi_{o^{l}}^{l}(v)\right)_{l} \in T_{o} \mathcal{O}$ and $c_{o}: h=\left(h^{l}\right) \in H \mapsto$ $\sum_{l} c_{o^{l}}^{l}\left(h^{l}\right)$.

A key point of our framework is the following stability result under combination: Proposition 3. If $M^{l}=\left(\mathcal{O}^{l}, H^{l}, \zeta^{l}, \xi^{l}, c^{l}\right), l=1 \cdots L$, are $C^{k}$-deformation modules of order $\ell$, then $\mathcal{C}\left(M^{l}, l=1 \cdots L\right)$ is a $C^{k}$-deformation module of order $\ell$. Furthermore, if each $M^{l}$ satisfies $U E C$, then $\mathcal{C}\left(M^{l}, l=1 \cdots L\right)$ also satisfies UEC.

Proof. It is clear that $\mathcal{C}\left(M^{l}, l=1 \cdots L\right)$ is a $C^{k}$-deformation module of order $\ell$. Suppose that each $M^{l}$ satisfies UEC. We define $W \doteq \prod_{l} V^{l}$ and

$$
\pi: w=\left(w_{1}, \ldots, w_{L}\right) \in W \mapsto \sum_{i} w_{i} \in C_{0}^{\ell}\left(\mathbb{R}^{d}\right) .
$$

Then the space $V \doteq \pi(W)$ can be equipped with the following norm: for $v \in V,|v|_{V}^{2}=$ $\inf \left\{\sum_{l}\left|v_{l}\right|_{V^{l}}^{2} \mid \pi\left(\left(v_{l}\right)_{l}\right)=v\right\}$, so that it is a Hilbert space continuously embedded in $C_{0}^{\ell}\left(\mathbb{R}^{d}\right)$. For any $o=\left(o^{l}\right) \in \mathcal{O}$ and $h=\left(h^{l}\right) \in H$, we have

$$
\left|\zeta_{o}(h)\right|_{V}^{2} \leq \sum_{l=1}^{L}\left|\zeta_{o^{l}}^{l}\left(h^{l}\right)\right|_{V_{l}}^{2} \leq \sum_{l=1}^{L} C_{l} c_{o^{l}}^{l}\left(h^{l}\right) \leq\left(\max _{1 \leq l \leq L} C_{l}\right) c_{o}(h),
$$


and then $\mathcal{C}\left(M^{l}, l=1 \cdots L\right)$ satisfies UEC.

Remark 4. Note that even if costs of elementary modules $M^{l}$ are given by $c_{o^{l}}^{l}\left(h^{l}\right)=$ $\left|\zeta_{o^{l}}^{l}\left(h^{l}\right)\right|_{V^{l}}^{2}$ as in our previous examples, in general (when $\pi$ is not one to one) the cost of the compound module is not the squared norm of the compound velocity field $\zeta_{o}(h)=$ $\sum_{l} \zeta_{o^{l}}^{l}$ in the global embedding RKHS V, i.e. $c_{o}(h)=\sum_{l}\left|\zeta_{o^{l}}^{l}\left(h^{l}\right)\right|_{V^{l}}^{2} \neq\left|\zeta_{o}(h)\right|_{V}^{2}$. Then in general $C>1$ and $c$ is not the pullback metric on $\mathcal{O} \times H$ of the metric on $\mathcal{O} \times V$. The cost $c_{o}(h)$ does not directly depend on the norm of the generated vector field $\zeta_{o}(h)$ but on its specific decomposition as a sum of elementary vector fields $\zeta_{o^{l}}^{l}\left(H^{l}\right)$. As we will consider configurations $(o, h)$ minimizing the cost $c_{o}(h)$ (for a given action $\xi_{o}\left(\zeta_{o}(h)\right)$ on the geometrical descriptor), different choices of cost $c^{l}$ can favour different decompositions for the same resulting vector field. Moreover, in practice, one can easily compute the cost of the compound module $c$ from the elementary costs $c^{l}$.

2.2. Large deformations. In this section, we show how large deformations can be generated from a given deformation module $M=(\mathcal{O}, H, \zeta, \xi, c)$ satisfying UEC. These large deformations are obtained as the integration of a trajectory of vector fields $v: t \in[0,1] \mapsto v_{t} \in V$ that are modular, meaning that at each time $t$ one can write $v_{t}=\zeta_{o_{t}}\left(h_{t}\right)$ with $\left(o_{t}, h_{t}\right) \in \mathcal{O} \times H$. During the integration of the trajectory we want the geometrical descriptor of the module to be transported by the flow and therefore, denoting $\varphi^{v}$ the flow of $v$ (see Proposition 1 ), that $v_{t}$ belongs to $\zeta_{o_{t}}(H)$, with $o_{t}=\varphi_{t}^{v}\left(o_{t=0}\right)$.

Definition 5. Let $a, b \in \mathcal{O}$. We denote $\Omega_{a, b}$ the set of measurable curves $t \mapsto$ $\left(o_{t}, h_{t}\right) \in \mathcal{O} \times H$ where $o_{t}$ is absolutely continuous (a.c.), starting from a and ending at $b$, such that for almost every $t \in[0,1], \dot{o}_{t}=\xi_{o_{t}}\left(v_{t}\right)$, where $v_{t} \doteq \zeta_{o_{t}}\left(h_{t}\right)$, and

$$
E(o, h) \doteq \int_{0}^{1} c_{o_{t}}\left(h_{t}\right) \mathrm{d} t<\infty .
$$

The quantity $E(o, h)$ is called the energy of $(o, h)$ and $\Omega_{a, b}$ is the set of controlled paths of finite energy starting at $a$ and ending at $b$.

If UEC is satisfied, we can build large deformations from these trajectories :

Proposition 4. Let us suppose that $M$ satisfies UEC. Let $(o, h) \in \Omega_{a, b}$ and for each $t, v_{t}=\zeta_{o_{t}}\left(h_{t}\right)$. Then $v \in L^{2}([0,1], V) \subset L^{1}$, the flow $\varphi^{v}$ exists, $h \in L^{2}([0,1], H)$ and for each $t \in[0,1], o_{t}=\phi_{t}^{v} . o_{0}$. We call the final diffeomorphism $\varphi_{t=1}^{v}$ a modular large deformation generated by a.

Proof. Let $(o, h) \in \Omega_{a, b}$ and $v: t \in[0,1] \mapsto v_{t} \doteq \zeta_{o_{t}}\left(h_{t}\right)$. From the UEC we get: $\int_{0}^{1}|v(t)|_{V}^{2} \mathrm{~d} t \leq C \int_{0}^{1} c_{o_{t}}\left(h_{t}\right) \mathrm{d} t<\infty$ since $(o, h) \in \Omega_{a, b}$. Then $v \in L^{2}([0,1], V) \subset$ $L^{1}([0,1], V) \subset L^{1}\left([0,1], C_{0}^{\ell}\left(\mathbb{R}^{d}\right)\right)$ and its flow $\varphi^{v}$ can be defined. As explained in Proposition 2, we can deduce that $o(t)=\varphi_{t}^{v} \cdot a$ for each $t$.

Let us now define, for $o \in \mathcal{O},\left\|c_{o}^{-1}\right\|:=\sup _{|h|_{H}=1} c_{o}(h)^{-1}$ (well defined as $H$ is of finite dimension). As $o \mapsto c_{o}$ and $t \mapsto o(t)=\varphi_{t}^{v} \cdot a$ are continuous, $\sup _{t}\left\|c_{o_{t}}^{-1}\right\|$ is finite and then $\int_{0}^{1}\left|h_{t}\right|_{H}^{2} \mathrm{~d} t \leq\left(\sup _{t} \| c_{o_{t}}^{-1}||\right) \int_{0}^{1} c_{o_{t}}\left(h_{t}\right) \mathrm{d} t<\infty$ so $h \in L^{2}([0,1], H)$.

Remark 5. A modular large deformation is parametrized by an initial value of geometrical descriptor $o_{t=0}$ and a trajectory of control $h \in L^{2}([0,1], H)$. 

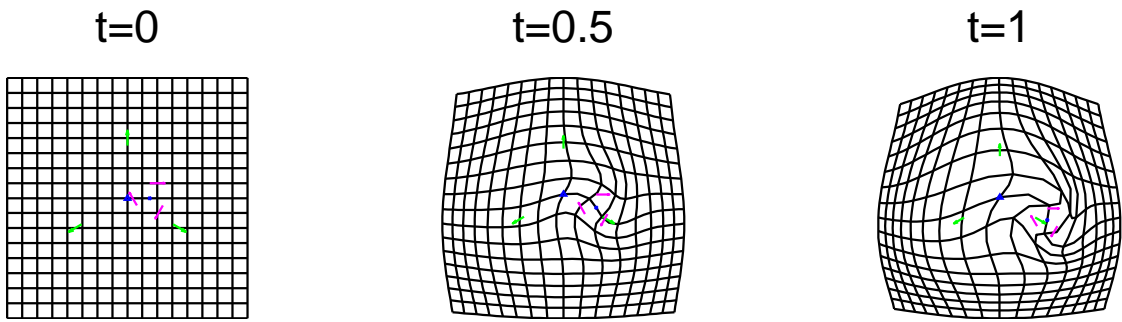

FIG. 2.5. Example of a large deformation. Geometrical descriptors are in blue (triangle for scaling, circle for rotation). Vectors are intermediate tools used to build vector fields (magenta for rotation and green for scaling)

2.3. Examples. We present here a list of possible deformation modules, which comes for the first case with an illustration of a modular large deformation. Most of these modules will be used in the numerical experiments in Section 5. Some modules will be presented only in dimension 2, but may be easily generalized in higher dimensions.

2.3.1. Constrained local transformations. In Section 2.1 .1 we presented the example of a deformation module generating vector fields that are always a local scaling at a fixed scale $\sigma$. In a more general setting, we can design deformation modules generating a particular type of local transformation by choosing different vectors $d_{j}$. Let us fix these vectors $d_{j}$ and therefore the corresponding local transformation $A$. Let us now build the deformation module that generates vector fields that are a sum of $P$ replications of $A$ at $P$ different locations. We set $\mathcal{O} \doteq\left(\mathbb{R}^{2}\right)^{P}, H \doteq \mathbb{R}^{P}$ and, for $o=\left(o_{i}\right)_{i} \in \mathcal{O}, \zeta_{o}: h=\left(h_{i}\right) \in H \mapsto \sum_{i=1}^{P} h_{i} \sum_{j=1}^{3} K_{\sigma}\left(z_{j}\left(o_{i}\right), \cdot\right) d_{j}$ (with points $z_{j}\left(o_{i}\right)$ defined in Section 2.1.1, Example 2), $\xi_{o}: v \in C_{0}^{\ell}\left(\mathbb{R}^{d}\right) \mapsto\left(v\left(o_{i}\right)\right)_{i} \in T \mathcal{O}$ (application of the vector field to each point), $c_{o}: h=\left(h_{i}\right)_{i} \in H \mapsto\left|\zeta_{o}(h)\right|_{V_{\sigma}}^{2}$. As for the deformation module generating one local scaling, it can be shown that $M=(\mathcal{O}, H, \zeta, \xi, c)$ is a deformation module satisfying UEC.

In Figure 2.5 we present an example of large deformation (see Proposition 4) generated by the combination of two deformation modules generating constrained local transforms: one generating a scaling at scale $\sigma_{1}$ and the second generating a rotation at scale $\sigma_{2}=\sigma_{1} / 3$. The deformation at each time is represented by the deformation of the grid. We can see that geometrical descriptors are transported by the global flow created by the two deformation modules. As its scale is smaller, the area of action of the local rotation is smaller than the one of the scaling. Then the area of influence of the local rotation is transported by the vector field created by the local scaling, while the geometrical descriptor of the local scaling is almost constant. We represent also in this figure intermediate tools $d_{j}$, which are recomputed at each time from the geometrical descriptors (centres of the scaling and rotation) and therefore are not transported by the flow. This example shows how complex modular large deformations can be naturally built from simple base modules, and how their mutual interaction during the integration of the trajectory is encoded in the compound deformation module.

2.3.2. Unconstrained local affine transformations. We present here the deformation module generating vector fields that are locally an affine deformation (at 
a fixed scale $\sigma$ ), without any other prior on the local deformation pattern. We explain here the construction in dimension 2 without loss of generality. For $o \in \mathbb{R}^{2}$ we define points $\left(z_{j}(o)\right)_{j}$ as in the previous case. We define spaces $\mathcal{O} \doteq \mathbb{R}^{2}, H \doteq\left(\mathbb{R}^{2}\right)^{3}$ (groups of 3 vectors of $\left.\mathbb{R}^{2}\right)$ and applications $\zeta:\left(o, h=\left(\alpha_{j}\right)\right) \in \mathcal{O} \times H \mapsto \sum_{j} K_{\sigma}\left(z_{j}(o), \cdot\right) \alpha_{j}$, $\xi_{o}: v \in C_{0}^{\ell}\left(\mathbb{R}^{2}\right) \mapsto v(o)$ and $c_{o}: h=\left(\alpha_{j}\right) \in H \mapsto\left|\zeta_{o}(h)\right|_{V_{\sigma}}^{2}$. Then $M=(\mathcal{O}, H, \zeta, \xi, c)$ is a $C^{k}$-deformation module satisfying UEC and generating vector fields that are unconstrained local transformations at scale $\sigma$. This example differs from the sum of translation in Section 2.1.1 (example 1) as the 3 centres of translations here are glued together. This example differs also from the previous one (Section 2.3.1), as here the directions $d_{j}$ are free to generate any local transform. These directions become then control parameters, whereas they were fixed in the previous example. Similarly to previous examples, local affine transforms may be combined into a deformation module creating a superimposition of $P$ unconstrained local transforms at different locations. This module class differs from the poly-affine framework [38] in that the neighbourhood which is affected by the local affine transformation is transported by the global deformation via the infinitesimal feedback action $\xi$.

2.3.3. Deformation module generating a sum of local translations. This deformation module enables us to see the construction of [14] as an instance of a deformation module and was detailed in Section 2.1.1, Example 1. The cost of this deformation module is $c:\left(o=\left(z_{i}\right), h=\left(\alpha_{i}\right)\right) \mapsto\left|\zeta_{o}(h)\right|_{V_{\sigma}}^{2}=\sum_{i, j} K_{\sigma}\left(z_{i}, z_{j}\right) \alpha_{i}^{T} \alpha_{j}$. Another possibility to generate sum of translations would be to consider the combination of modules creating one translation, such that the cost would be $\sum_{i}\left|K_{\sigma}\left(\cdot, z_{i}\right) \alpha_{i}\right|_{V_{\sigma}}^{2}=$ $\sum_{i}\left|\alpha_{i}\right|^{2}$. The first case is more interesting because minimizing the cost tends to separate centres $z_{i}$ and then forces local translations of the same scale to act everywhere it is needed, while in the second case several ones could converge to the same location, making it redundant.

2.3.4. Example of combination: a multi-scale sum of local translations. Let us fix $P$ scales $\sigma_{l}$ and for each $l$ a number of translations $D_{l}$. We present here the construction of the deformation module that generates vector fields that are a sum of multi-scale local translations, with $D_{l}$ translations for each scale $\sigma_{l}$. For each $l$ can be built, as defined in Section 2.1.1 (first example), a module $M^{l}$ generating vector fields that are a sum of $D_{l}$ local translations acting at scale $\sigma_{l}$. The multi-scale module $M$ is then the combination of these modules $M^{l}$ (see Section 2.1.3). In particular the vector field created by geometrical descriptor $o=\left(z_{j}^{l}\right) \in \mathcal{O}=\Pi_{l}\left(\left(\mathbb{R}^{d}\right)^{D_{l}}\right)$ and control $h=\left(\alpha_{j}^{l}\right) \in H=\Pi_{l}\left(\left(\mathbb{R}^{d}\right)^{D_{l}}\right)$ is $\zeta_{o}(h)=\sum_{l} \sum_{j} K_{\sigma_{l}}\left(z_{j}^{l}, \cdot\right) \alpha_{j}^{l}$. It is clear here that, unlike in the framework of [36] where vector fields are generated based on a sum of Gaussian kernels, centers of local translations are different for each scale. This property is kept for optimal trajectories (minimising the cost) as we force these points to be different at the initial time.

The cost is, for $o=\left(z_{j}^{l}\right) \in \mathcal{O}$ and $h=\left(\alpha_{j}^{l}\right) \in H, c_{o}(h)=\sum_{l} \sum_{j} K_{\sigma_{l}}\left(z_{j}^{l}, z_{j^{\prime}}^{l}\right) \alpha_{j}^{l T} \alpha_{j^{\prime}}^{l}$. Even though this cost is similar to the framework of kernel bundle presented in [42], the control variables which are optimized in our framework are only the vectors of local translations $h=\left(\alpha_{j}^{l}\right) \in H$ and not the global vector field. As a consequence even for optimal trajectories, the decomposition of the vector field in a sum of local translations acting at different scales and centered at different points is preserved. On the other hand, as shown in [11], in the kernel bundle framework [42] optimal trajectories coincide with these of the sum of kernel's framework and then authors introduced a sparse penalty in [40] to address this issue. Here we do not need to 
introduce such a sparse penalty because the control variable that we optimize is not the vector field but only the vectors of the translations: we impose the form of the vector field as a prior.

2.3.5. Deformation module generating a sum of local translations with a prior on the direction. In the previous examples of deformation modules based on local translations (Sections 2.3.3 and 2.3.4), the direction and magnitude of the translation vector were considered as control parameters. Therefore, during the integration of the flow, the direction of the translation at each time needs to be determined as an optimal solution for a given criterion. By contrast, we may want to update the direction of the translation during deformation by using a prior rule, thus considering the direction no more as a control parameter but instead as a geometrical descriptor. In this case, only the magnitude of the translation vector is considered as a control parameter. We give two examples of such modules for translations acting at scale $\sigma$.

Translation with constant direction. In this example, the translation vector is kept constant during integration, meaning that the direction of the translation is linked to a fixed background and not to the shape that is deformed. We denote by $u \in \mathbb{R}^{d}$ this direction, and set $\mathcal{O} \doteq \mathbb{R}^{d}, H \doteq \mathbb{R}$ (the control is scalar) and for $(z, u) \in \mathbb{R}^{d} \times \mathbb{R}^{d}$, $h \in \mathbb{R}, v \in C_{0}^{1}\left(\mathbb{R}^{d}\right), \zeta_{o}(h) \doteq K_{\sigma}(z, \cdot) h u, \xi_{o}(v)=(v(z), 0)$ and $c_{o}(h)=\left|\zeta_{o}(h)\right|_{V_{\sigma}}^{2}=h^{2}$. The geometrical descriptors are the base point of the translation vector, which is transported by the deformation flow, and the direction $u$, which is kept constant. This deformation module can be generalized to the deformation module generating vector fields that are a sum of $P$ local translations at scale $\sigma$ with fixed directions $u_{i}$ by setting $\mathcal{O} \doteq\left(\mathbb{R}^{d}\right)^{P} \times\left(\mathbb{R}^{d}\right)^{P}, H \doteq \mathbb{R}^{P}$ (the control is made of $P$ scalars), $V \doteq V_{\sigma}$ and for $o=\left(z_{i}, u_{i}\right) \in \mathcal{O}, h=\left(h_{i}\right) \in H, v \in C_{0}^{1}\left(\mathbb{R}^{d}\right), \zeta_{o}(h) \doteq \sum_{i} K_{\sigma}\left(z_{i}, \cdot\right) h_{i} u_{i}$, $\xi_{o}(v)=\left(v\left(z_{i}\right), u_{i}\right)$ and $c_{o}(h)=\left|\zeta_{o}(h)\right|_{V_{\sigma}}^{2}$. This deformation module satisfies also UEC and will be used in examples of Section 5.1.

Translation with direction updated by adjoint action. This example is the same as the previous one, except that we change the update rule for the direction $u$ of the translation. The infinitesimal action on geometrical descriptors writes now $\xi_{o}(v)=\left(v(z),-d v_{z}^{T} u\right)$ (with $o=(z, u)$ ). The integration of this infinitesimal action gives the action of the deformation $\phi$ on the geometrical descriptors $o$ as $(\phi, o=$ $(z, u)) \mapsto\left(\phi(z),\left(\mathrm{d} \phi_{\phi(x)}^{-1}\right)^{T} u\right)$, where the second part is the so-called adjoint action. If one considers the direction $u$ as the normal to an infinitesimal surface patch, then $\left.\left(\mathrm{d} \phi_{\phi(x)}^{-1}\right)^{T} u\right)$ is the direction of the normal of the deformed surface patch. If we consider a compound module combining this deformation module with others, the direction of the translation at integration time $t$ will only depend on the generated global diffeomorphism $\phi$ and the direction $u$ at time $t=0$. As above, the combination of $P$ of these modules also satisfy UEC. They will be used in examples in Section 5.2.

2.3.6. Silent modules. We present here a last and important example of deformation modules: modules generating a vector field that is always null. For a choice of $\mathcal{O}$, a $C^{k}$-shape space of order $\ell$ with its infinitesimal action $\xi$, we set $H \doteq\{0\}$ (null space for the controls) and for $o \in \mathcal{O}, \zeta_{o}: h \in H \mapsto 0, c_{o}: h \in H \mapsto 0$. Then $M=(\mathcal{O}, H, \zeta, \xi, c)$ defines a $C^{k}$-deformation module of order $\ell$ satisfying UEC, which will be referred to as the silent deformation module induced by shape space $\mathcal{O}$. For instance, if $\mathcal{O}$ is the shape space made up of a collection of points as in the examples of landmarks (Section 2.1.1), then these points, considered as a silent module, will feel the velocity field generated by active modules located around them, and move accordingly, but will not contribute to this velocity field. In contrast to this 
notion of silent deformation module, we will say that the other deformation modules (generating vector fields non necessarily equal to zero) are active. As we will detail in section 3.2 .2 , the silent deformation modules are particularly interesting to study the case of a matching problem where the active deformation modules used to generate the large deformation have geometrical descriptors different from the shape data.

3. Sub-Riemannian setting. We explain here how modular large deformations may be used to provide shape spaces with a sub-Riemannian metric, and therefore define a distance between shapes.

We consider a $C^{k}$-deformation module $M=(\mathcal{O}, H, \zeta, \xi, c)$ of order $\ell \in \mathbb{N}^{*}$. A geometrical descriptor of the module is a "shape" in the shape space $\mathcal{O}$ and, intuitively, the orbit of this geometrical descriptor under the action of a regular group of diffeomorphisms forms a Riemannian manifold. If one considers only the diffeomorphisms which result from the integration of a modular velocity field (generated by the module $M)$, then one provides this Riemannian manifold with a sub-Riemannian structure (Section 3.1). This construction allows the definition of a sub-Riemannian distance and of optimal trajectories between two geometrical descriptors in the shape space $\mathcal{O}$ (Section 3.2).

In Section 3.2.2 we study the practical case where we want to study shape data belonging to a common shape space $\mathcal{F}$ via deformations generated by a deformation module whose space of geometrical descriptors is different of $\mathcal{F}$.

3.1. A sub-Riemannian structure on $\mathcal{O}$. We suppose that $M$ satisfies UEC, and we fix $V$ a space of vector fields and $C$ a positive constant such that $V$ is continuously embedded in $C_{0}^{\ell+k}\left(\mathbb{R}^{d}\right)$ and for all $o \in \mathcal{O}$ and for all $h \in H, \zeta_{o}(h) \in V$ and $\left|\zeta_{o}(h)\right|_{V}^{2} \leq C c_{o}(h)$. We now use the notion of continuous sub-Riemannian structure on a manifold, following the definition given in [4].

Definition 6. Let $\mathcal{M}$ be a manifold of finite dimension. We define a continuous sub-Riemannian structure on $\mathcal{M}$ as a triple $(\mathcal{E}, g, \rho)$, where $: \mathcal{E} \rightarrow \mathcal{M}$ is a smooth vector bundle on $\mathcal{M}$ endowed with a smooth Riemannian metric $g$, and $\rho: \mathcal{E} \rightarrow T M$ a continuous vector bundle morphism.

The composition of the field generator $\zeta$ with the infinitesimal action $\xi$ yields a continuous vector bundle morphism $\rho:(o, h) \in \mathcal{O} \times H \mapsto\left(o, \rho_{o}(h)=\xi_{o} \circ \zeta_{o}(h)\right) \in T \mathcal{O}$. Moreover, the cost $c$ induces a smooth Riemannian metric $g$ on the vector bundle $\mathcal{O} \times H$. Then $(\mathcal{O} \times H, g, \rho)$ defines a sub-Riemannian manifold, which we will denote by $\mathcal{O}_{H}$. This structure is the key to define trajectories of modular deformations. At each shape $o \in \mathcal{O}$, the space $\zeta_{o}(H)$ is the space of vector fields that can be generated by $o$. The horizontal space $\rho_{o}(H)=\xi_{o}\left(\zeta_{o}(H)\right)$ is the set of tangent vectors of $T_{o} \mathcal{O}$ that can be obtained by the action of the geometrical descriptor $o$ on itself.

Remark 6. Note that the dimension of the horizontal space at $o \in \mathcal{O}$, namely the rank of the sub-Riemannian structure at $o \in \mathcal{O}$, may depend on o. For instance, let us consider the deformation module $M=(\mathcal{O}, H, \zeta, \xi, c)$ obtained by combining a module $M^{1}=\left(\mathcal{O}^{1}, H^{1}, \zeta^{1}, \xi^{1}, c^{1}\right)$ generating a local scaling in $\mathbb{R}^{2}$ at scale $\sigma$ (see Section 2.1.1, second example) and a module $M^{2}=\left(\mathcal{O}^{2}, H^{2}, \zeta^{2}, \xi^{2}, c^{2}\right)$ generating a sum of three local translations in $\mathbb{R}^{2}$ at scale $\sigma$ (see Section 2.1.1, first example). For each geometrical descriptor $o=\left(o^{1}, o^{2}=\left(z_{i}\right)_{1 \leq i \leq 3}\right) \in \mathcal{O}^{1} \times \mathcal{O}^{2}=\mathbb{R}^{2} \times\left(\mathbb{R}^{2}\right)^{3}$ and each control $h=\left(h^{1}, h^{2}=\left(\alpha_{i}\right)_{1 \leq i \leq 3}\right) \in H^{1} \times H^{2} \stackrel{\mathbb{R}}{=} \times\left(\mathbb{R}^{2}\right)^{3}$, the generated vector field is $\zeta(o, h)=h^{1} \sum_{j} K\left(z_{j}\left(o^{1}\right), \cdot\right) d_{j}\left(o^{1}\right)+\sum_{i} K\left(z_{i}, \cdot\right) \alpha_{i}$ with $z_{j}\left(o^{1}\right)$ and $d_{j}\left(o^{1}\right)$ defined in Section 2.1.1, Example 2. Then in the particular case where $o^{1}$ and $o^{2}=\left(z_{i}\right)_{i}$ are such that $z_{i}=z_{i}\left(o^{1}\right)$ for $i \in\{1,2,3\}, \operatorname{dim}\left(\xi_{o}\left(\zeta_{o}(H)\right)\right)=2 \times 3=6$ while in other cases 
$\operatorname{dim}\left(\xi_{o}\left(\zeta_{o}(H)\right)\right)=2 \times 3+1=7$.

The cost $c$ equips the vector bundle $\mathcal{O} \times H$ with a metric $g$, which may be used to derive a metric $g^{\mathcal{O}}$ on $\mathcal{O}_{H}$ : if $\delta o, \delta o^{\prime} \in \rho_{o}(H)$, let $h, h^{\prime} \in \operatorname{Ker}\left(\rho_{o}\right)^{\perp}$ (orthogonal for the metric $g$ on $\mathcal{O} \times H$ ) such that $\rho_{o}(h)=\delta o$ and $\rho_{o}\left(h^{\prime}\right)=\delta o^{\prime}$, then $g_{o}^{\mathcal{O}}\left(\delta o, \delta o^{\prime}\right) \doteq g_{o}\left(h, h^{\prime}\right)$. Note that for $\delta o \in \rho_{o}(H),|\delta o|_{o}^{2} \doteq g_{o}^{\mathcal{O}}(\delta o, \delta o)=\inf \left\{c_{o}(h) \mid\right.$ $\left.\rho_{o}(h)=\delta o, h \in H\right\}$. This sub-Riemannian metric $g^{\mathcal{O}}$ will be the one used to build the new distance on $\mathcal{O}$.

Definition 7. [1] Let $\mathcal{M}$ be a smooth manifold of finite dimension equipped with a continuous sub-Riemannian structure $(\mathcal{E}, g, \rho)$. A horizontal system is a curve $t \in[0,1] \mapsto(q(t), u(t)) \in \mathcal{E}$ such that $t \in[0,1] \mapsto u(t) \in \mathcal{E}_{q(t)}$ is of class $L^{2}$ (ie $\left.\int_{0}^{1} g_{q(t)}(u(t), u(t)) \mathrm{d} t<\infty\right)$, and its projection $: t \in[0,1] \mapsto q(t)$ is absolutely continuous and satisfies for almost every $t \in[0,1], \dot{q}(t)=\rho_{q(t)} u(t)$. A horizontal curve is the projection $q(\cdot)$ to $\mathcal{M}$ of a horizontal system.

Remark 7. For $a, b \in \mathcal{O}$, the space $\Omega_{a, b}$ (see Definition 5) is exactly the set of horizontal system connecting $a$ and $b$.

Definition 8. [1] Let $o:[0,1] \longrightarrow \mathcal{O}$ be a horizontal curve, we define its subRiemannian length:

$$
l(o)=\int_{0}^{1}\left|\dot{o}_{t}\right|_{o_{t}} \mathrm{~d} t=\int_{0}^{1} \sqrt{g_{o_{t}}^{\mathcal{O}}\left(\dot{o_{t}}, \dot{o_{t}}\right)} \mathrm{d} t .
$$

For $a, b \in \mathcal{O}$ we can then define the sub-Riemannian distance

$$
D(a, b)=\inf \left\{l(o) \mid \exists h:(o, h) \in \Omega_{a, b}\right\} .
$$

Proposition 5. As $M$ satisfies $U E C$, the sub-Riemannian distance $D$ is a true distance on $\mathcal{O}$.

Proof. It is clear that $D$ is a pseudo-distance, so we only need to show that if $D(a, b)=$ 0 then $a=b$. We will use a result proved in [6]: if we set for $\phi \in \operatorname{Diff}_{0}^{\ell}\left(\mathbb{R}^{d}\right)$, $d(I d, \phi) \doteq \inf \left\{\int_{0}^{1}\left|v_{t}\right|_{V} \mathrm{~d} t \mid \forall t v_{t} \in V\right.$ and $\left.\varphi_{t=1}^{v}=\phi\right\}$ (we remind that $V$ is the Hilbert space of vector fields defined in UEC satisfied by $M)$ and for $a, b \in \mathcal{O}$, $\operatorname{dist}(a, b)=$ $\inf \{d(I d, \phi) \mid \phi \cdot a=b\}$ then dist defines a distance (taking its value in $[0,+\infty])$.

Let $a, b \in \mathcal{O}$ such that $D(a, b)=0$, then there exists $\left(o^{n}\right)_{n \in \mathbb{N}}$ such that for each $n$ there exists $h^{n}$ such that $\left(o^{n}, h^{n}\right) \in \Omega_{a, b}$ and $l\left(o^{n}\right) \longrightarrow 0$. By choosing $h^{n}$ the minimal control of $o^{n}$ (see [1]), we also have $l\left(o^{n}, h^{n}\right) \longrightarrow 0$. Yet, from the UEC, for any $n$, $\operatorname{dist}(a, b) \leq \int_{0}^{1}\left|\zeta_{o_{t}^{n}}\left(h_{t}^{n}\right)\right|_{V} \mathrm{~d} t \leq \sqrt{C} \int_{0}^{1} \sqrt{c_{o_{t}^{n}}\left(h_{t}^{n}\right)} \mathrm{d} t=\sqrt{C} l\left(o^{n}, h^{n}\right) \longrightarrow 0$. Then $a=b$.

3.2. Optimal trajectories. As the quantity $l(o)=\int_{0}^{1}\left|\dot{o}_{t}\right|_{o_{t}} \mathrm{~d} t$ is not convenient to study, in order to characterize the sub-Riemannian distance and the minimizing trajectories we first need to link them with minimizers of the energy $E(o, h)=$ $\int_{0}^{1} c_{o_{t}}\left(h_{t}\right) \mathrm{d} t$. This is done using the following proposition:

Proposition 6. [1] Let $a$ and $b$ be in $\mathcal{O}$, then

$$
D(a, b)=\inf \left\{E(o, h) \mid(o, h) \in \Omega_{a, b}\right\}
$$

and the cost $c_{o}(h)$ is constant along minimizers. 
3.2.1. Existence of optimal trajectories. Proposition 6 shows that calculating the distance $D(a, b)$ between two elements $a, b$ of $\mathcal{O}$ amounts to looking for a horizontal system minimizing the energy $E$, which is easier to study. Therefore, in the next paragraph, we characterize the horizontal system minimizing $E$. We need here to restrain ourselves to a certain type of shapes, obtained through an adaptation of a definition given by S. Arguillère in [6].

Definition 9. An element o of $\mathcal{O}$, is said to be of compact support if there exists a compact set $K$ in $\mathbb{R}^{d}$ such that for all $\phi \in D_{0}^{\ell}\left(\mathbb{R}^{d}\right), \phi \cdot$ o only depends on $\phi_{\mid K}$ and $\phi \in D_{K}^{\ell} \mapsto \phi \cdot o$ is continuous with $D_{K}^{\ell}:=\left\{\phi_{\mid K} \mid \phi \in D_{0}^{\ell}\left(\mathbb{R}^{d}\right)\right\}$ equipped with the distance deduced from the norm on $\left\{v_{\mid K} \mid v \in C_{0}^{\ell}\left(\mathbb{R}^{d}\right)\right\}:|v|_{\ell, K}=\sup \left\{\left|\frac{\partial^{\ell_{1}+\cdots+\ell_{d}} v(x)}{\partial x_{1}^{\ell_{1} \cdots x_{d}^{\ell_{d}}}}\right| \mid\right.$ $\left.x \in K,\left(\ell_{1}, \cdots, \ell_{d}\right) \in \mathbb{N}^{d}, \ell_{1}+\cdot+\ell_{d} \leq \ell\right\}$.

Remark 8. Examples presented in Section 2.3 correspond to geometrical descriptors of compact support.

Lemma 1. Let $o \in \mathcal{O}$ be of compact support and let $K$ be a compact set of $\mathbb{R}^{d}$ such that for all $\phi$ of $D_{0}^{\ell}\left(\mathbb{R}^{d}\right) \phi \cdot o$ only depends on $K$ and $\phi \in D_{K}^{\ell} \mapsto \phi \cdot o$ is continuous. Then for each $\phi \in D_{0}^{\ell}\left(\mathbb{R}^{d}\right), \phi \cdot o$ is of compact support and $\psi \in D_{0}^{\ell}\left(\mathbb{R}^{d}\right) \mapsto \psi \cdot(\phi \cdot o)$ only depends on the compact set $\phi(K)$.

Proof. Let $\phi, \psi$ and $\psi^{\prime}$ be elements of $D_{0}^{\ell}\left(\mathbb{R}^{d}\right)$ such that $\psi_{\mid \phi(K)}=\psi_{\mid \phi(K)}^{\prime}$. Then $\psi \cdot(\phi \cdot o)=(\psi \circ \phi) \cdot o=\left(\psi^{\prime} \circ \phi\right) \cdot o$ because $\psi \circ \phi_{\mid K}=\psi^{\prime} \circ \phi_{\mid K}$. Then $\psi \cdot(\phi \cdot o)=\psi^{\prime} \cdot(\phi \cdot o)$ and we conclude that for all $\psi \in D_{0}^{\ell}\left(\mathbb{R}^{d}\right), \psi \cdot(\phi \cdot o)$ only depends on $\phi(K)$. Besides, from Faà di Bruno's formula one gets that for any $\phi \in D_{0}^{\ell}\left(\mathbb{R}^{d}\right)$, there exists a constant $C_{|\phi|_{\ell, K}}$ such that for each $\psi, \psi^{\prime}$ in $D_{0}^{\ell}\left(\mathbb{R}^{d}\right),\left|\psi \circ \phi-\psi^{\prime} \circ \phi\right|_{\ell, K} \leq C_{|\phi|_{\ell, K}}\left|\psi-\psi^{\prime}\right|_{\ell, \phi(K)}$. Then for $\phi \in D_{0}^{\ell}\left(\mathbb{R}^{d}\right), \psi \in D_{0}^{\ell}\left(\mathbb{R}^{d}\right) \mapsto \psi \cdot(\phi \cdot o)$ is continuous and therefore $\phi \cdot o$ is of compact support.

Let $a$ and $b$ be two geometrical descriptors of compact support, we prove here the existence of minimizers of the energy (if $\Omega_{a, b}$ is non-empty), and therefore of trajectories reaching sub-Riemannian distance $D$.

Theorem 1. Let $M=(\mathcal{O}, H, \zeta, \xi, c)$ be a deformation module satisfying UEC and let $a, b$ in $\mathcal{O}$ such that $\Omega_{a, b}$ (set of finite energy horizontal systems $(o, h)$ such that $o$ starts at $a$ and ends at $b$ ) is non-empty. Then the energy $E$ (see Definition 5$)$ reaches its minimum on $\Omega_{a, b}$.

Proof. Let $\left(o^{n}, h^{n}\right)$ be a minimizing sequence of $E$ in $\Omega_{a, b}$ and let, for each $n, \varphi^{n}$ be the flow associated with $\left(o^{n}, h^{n}\right)$ as defined in Proposition 4: $\varphi^{n}=\varphi^{v_{n}}$ with $v_{n}=\zeta_{o^{n}}\left(h^{n}\right)$. Since $\int_{0}^{1}\left|v_{t}^{n}\right|_{V}^{2} \mathrm{~d} t \leq C E\left(o^{n}, h^{n}\right)$ (from UEC), the sequence $\left(v_{n}\right)_{n}$ is bounded in $L^{2}\left([0,1], \mathbb{R}^{d}\right)$ so up to the extraction of a subsequence we can assume that $v^{n}$ converges weakly to $v^{\infty} \in L^{2}\left([0,1], \mathbb{R}^{d}\right)$. Let us define $\varphi^{\infty}$ the flow of $v^{\infty}$. As $a$ is of compact support, there exists $K$ compact of $\mathbb{R}^{d}$ such that for all $\phi \in D_{0}^{\ell}\left(\mathbb{R}^{d}\right), \phi \cdot a$ only depends on $\phi_{\mid K}$. Moreover, as $K$ is compact, [16] shows that $\sup _{(t, x) \in[0,1] \times K} \mid \phi_{t}^{n}(x)-$ $\phi_{t}^{\infty}(x) \mid \longrightarrow 0$ so that, as $\phi \in D_{K}^{\ell} \mapsto \phi \cdot a$ is continuous, $o^{n}=\phi^{n} \cdot a$ converges to $o^{\infty}=\phi^{\infty} \cdot a$ uniformly on $[0,1]$. Therefore there exists a compact set $L$ of $\mathcal{O}$ such that for all $t, o_{t}^{\infty} \in L$ and for all $n, o_{t}^{n} \in L$. Then $\sup _{o \in L}\left\|c_{o}^{-1}\right\|$ (see proof of Proposition 4) is finite and for each $n: \int_{0}^{1}\left|h^{n}(t)\right|_{H}^{2} \mathrm{~d} t \leq \sup _{o \in L}\left\|c_{o}^{-1}\right\| E\left(o^{n}, h^{n}\right)$. Therefore $h^{n}$ is bounded in $L^{2}([0,1], H)$ so up to the extraction of a subsequence we can assume that $h^{n}$ converges weakly to $h^{\infty} \in L^{2}([0,1], H)$. Let us show that 
$\left(o^{\infty}, h^{\infty}\right) \in \Omega_{a, b}$. Let $w \in L^{2}([0,1], V)$, we have

$$
\begin{aligned}
\left|\int_{0}^{1}\left\langle v_{t}^{\infty}-\zeta_{o_{t}^{\infty}}\left(h_{t}^{\infty}\right), w_{t}\right\rangle_{V} \mathrm{~d} t\right| \leq & \left|\int_{0}\left\langle v_{t}^{\infty}-\zeta_{o_{t}^{n}}\left(h_{t}^{n}\right), w_{t}\right\rangle_{V} \mathrm{~d} t\right| \\
& +\left|\int_{0}^{1}\left\langle\zeta_{o_{t}^{n}}\left(h_{t}^{n}\right)-\zeta_{o_{t}^{\infty}}\left(h_{t}^{n}\right), w_{t}\right\rangle_{V} \mathrm{~d} t\right| \\
& +\mid \int_{0}^{1}\left\langle\zeta_{o_{t}^{\infty}}\left(h_{t}^{n}\right)-\zeta_{o_{t}^{\infty}}\left(h_{t}^{\infty}\right), w_{t}\right\rangle_{V} \mathrm{~d} t .
\end{aligned}
$$

As $\zeta_{o^{n}}\left(h^{n}\right)$ converges weakly to $v^{\infty}$ the first term tends to 0 . In the same way, $h \in L^{2}([0,1], H) \mapsto \int_{0}^{1}\left\langle\zeta_{o_{t}^{\infty}}\left(h_{t}\right), w_{t}\right\rangle_{V} \mathrm{~d} t$ is continuous since, as $\zeta$ is of class $C^{1}$ with respect to $o, o \in L \mapsto \zeta_{o} \in L(H, V)$ is bounded on $L$ (which contains $o_{t}^{\infty}$ for all $t$ ). Then as $h^{n}$ weakly converges to $h^{\infty}, \int_{0}^{1}\left\langle\zeta_{o_{t}^{\infty}}\left(h_{t}^{n}\right)-\zeta_{o_{t}^{\infty}}\left(h_{t}^{\infty}\right), w_{t}\right\rangle_{V} \mathrm{~d} t$ tends to 0 . Therefore

$$
\begin{aligned}
\left|\int_{0}^{1}\left\langle v_{t}^{\infty}-\zeta_{o_{t}^{\infty}}\left(h_{t}^{\infty}\right), w_{t}\right\rangle_{V} \mathrm{~d} t\right| & \leq \limsup \left|\int_{0}^{1}\left\langle\left(\zeta_{o_{t}^{n}}-\zeta_{o^{\infty}}\right)\left(h_{t}^{n}\right), w_{t}\right\rangle \mathrm{d} t\right| \\
& \leq \lim \sup \left(\int_{0}\left|w_{t}\right|_{V}^{2} \mathrm{~d} t \int_{0}^{1}\left\|\zeta_{o_{t}^{n}}-\zeta_{o_{t}^{\infty}}\right\|^{2}\left|h_{t}^{n}\right|^{H} \mathrm{~d} t\right)^{1 / 2} \\
& =0,
\end{aligned}
$$

since $h^{n}$ is bounded in $L^{2}([0,1], H)$ and, as $o^{n}$ uniformly converges to $o^{\infty}$, $\| \zeta_{o_{t}^{n}}-$ $\zeta_{o_{t}^{\infty}} \| \longrightarrow 0$. Since $w$ is arbitrary, $v^{\infty}=\zeta_{o^{\infty}}\left(h^{\infty}\right)$ and $\dot{o}_{t}^{\infty}=\xi_{o_{t}^{\infty}}\left(v_{t}^{\infty}\right)$ so that $\left(o^{\infty}, h^{\infty}\right) \in \Omega_{a, b}$. We now need to show that $E\left(o^{\infty}, h^{\infty}\right)=\lim E\left(o^{n}, h^{n}\right)$. Since $h \mapsto$ $\int_{0}^{1} c_{o_{t}^{\infty}}(h) \mathrm{d} t$ is continuous and convex $\int_{0}^{1} c_{o_{t}^{\infty}}\left(h_{t}^{\infty}\right) \mathrm{d} t \leq \lim \inf \int_{0}^{1} c_{o_{t}^{\infty}}\left(h_{t}^{n}\right) \mathrm{d} t$. Moreover since $c$ is a continuous metric, there exists $C: o \mapsto C_{o}$ continuous such that $c_{o}(h)=$ $\left(C_{o} h \mid h\right)_{H^{\star}}$, so that $\left|\int_{0}^{1}\left(c_{o_{t}^{\infty}}\left(h_{t}^{n}\right)-c_{o_{t}^{n}}\left(h_{t}^{n}\right)\right) \mathrm{d} t\right| \leq\left(\sup _{t}|| C_{o_{t}^{\infty}}-C_{o_{t}^{n}}||\right) \int_{0}^{1}\left|h_{t}^{n}\right|_{H}^{2} \mathrm{~d} t \longrightarrow 0$. Then we obtain $\int_{0}^{1} c_{o_{t}^{\infty}}\left(h_{t}^{\infty}\right) \mathrm{d} t \leq \liminf \int_{0}^{1} c_{o_{t}^{n}}\left(h_{t}^{n}\right) \mathrm{d} t$ and $E\left(o^{\infty}, h^{\infty}\right)=\lim E\left(o^{n}, h^{n}\right)$.

3.2.2. The matching problem in practice. In practice, one wants to study the differences between shapes belonging to a common shape space $\mathcal{F}$ via a user defined deformation module $M^{1}=\left(\mathcal{O}^{1}, H^{1}, \zeta^{1}, \xi^{1}, c^{1}\right)$ independent from the deformation module. We detail here this practical case. We can use the previous construction by introducing the silent deformation module $M^{2}=\left(\mathcal{F}, H^{2}, \zeta^{2}, \xi^{2}, c^{2}\right)$ induced by shape space $\mathcal{F}$ (see Section 2.3.6) and its combination $M=(\mathcal{O}, H, \zeta, \xi, c)$ with $M^{1}$ (see Section 2.1.3). The deformation module $M$ satisfies UEC and for $a=(o, f) \in \mathcal{O}=\mathcal{O}^{1} \times \mathcal{F}, h=\left(h^{1}, 0\right) \in H$, the application to $a$ of the vector field generated by $a$ and the control $h$ is

$$
\xi_{o} \circ \zeta_{o}(h)=\xi_{o} \circ \zeta_{o^{1}}^{1}\left(h^{1}\right)=\left(\xi_{o^{1}}^{1} \circ \zeta_{o^{1}}\left(h^{1}\right), \xi_{f}^{2} \circ \zeta_{o^{1}}\left(h^{1}\right)\right) .
$$

As previously, the cost $c$ allows to build a sub-Riemannian structure on $\mathcal{O} \times \mathcal{F}$ and horizontal trajectories are such that the $\mathcal{F}$ component is transported by the vector field generated by the component in $\mathcal{O}$. Note that the large deformation generated by this compound module are the same as those generated by $M^{1}$, but that optimal trajectories are different.

Let $a$ and $b$ be two shapes of $\mathcal{O}=\mathcal{O}^{1} \times \mathcal{F}$ of compact support. We distinguish in these shapes the silent component (belonging to $\mathcal{F}$ ) and the active one (belonging to $\mathcal{O}): a=\left(o^{1}, f^{1}\right)$ and $b=\left(o^{2}, f^{2}\right)$. We consider now the practical case where the target shape $b$ does not derive from the source shape $a$ by the action of a modular diffeomorphism. Instead, we propose to find the "optimal" shape $\hat{b}=\left(\hat{o}^{2}, \hat{f}^{2}\right)$ in the orbit of $a$, i.e. such that $\hat{b}=\phi . a$, so that the deformed silent component $\hat{f}^{2}=\phi \cdot f^{1}$ 
falls as close as possible to $f^{2}$ in the sense of a measure $\mu$. Determining this optimal shape $\hat{b}=\left(\hat{o}^{2}, \hat{f}^{2}\right)$ amounts to solving an inexact matching problem by minimizing the following functional (see [21]):

$$
J_{a, b}(h)=\frac{1}{\sigma^{2}} \mu\left(f_{t=1}, f^{2}\right)+\int_{0}^{1} c_{o_{t}}\left(h_{t}\right) \mathrm{d} t=\frac{1}{\lambda^{2}} \mu\left(f_{t=1}, f^{2}\right)+\int_{0}^{1} c_{o_{t}^{1}}^{1}\left(h_{t}^{1}\right) \mathrm{d} t,
$$

with $o_{t=0}=a, b=\left(o^{2}, f^{2}\right), o=\left(o^{1}, f\right), h=\left(h^{1}, 0\right), \dot{o}=\left(\dot{o}^{1}, \dot{f}\right)=\left(\xi_{o^{1}}^{1} \circ \zeta_{o^{1}}^{1}\left(h^{1}\right), \xi_{f}^{2} \circ\right.$ $\left.\zeta_{o^{1}}^{1}\left(h^{1}\right)\right)$ and $\lambda$ a scalar (chosen so that the two terms in the sum are of the same order of magnitude). The function $\mu$ is called the data attachment function as it measures how close to the target shape the source shape is transported..

A trajectory $o$ of $\mathcal{O}$ starting at $a$ such that there exists $h \in L^{2}([0,1], H)$ so that $(o, h)$ is a horizontal system (see Definition 7$)$ and $h$ minimizes $J_{a, b}$, will be called an optimal trajectory starting at $a$. The existence of such optimal trajectories is ensured by Theorem 1. Besides they can be well characterized via the next result, which we prove following the idea of the proof of [4].

Theorem 2. We recall that $M=(\mathcal{O}, H, \zeta, \xi, c)$ is a $C^{k}$-deformation module of order $C^{\ell}$ satisfying $U E C$ with $k, l \geq 1$. We suppose that the data attachment function $\mu$ is $C^{1}$. If $h \in L^{2}([0,1], H)$ minimizes functional $J_{a, b}$ then there exists a path $\eta: t \in$ $[0,1] \longrightarrow \eta_{t} \in T_{o_{t}}^{*} \mathcal{O}$ such that with

$$
\mathcal{H}:(o, \eta, h) \in T^{*} \mathcal{O} \times H \mapsto\left(\eta \mid \xi_{o}\left(\zeta_{o}(h)\right)\right)-\frac{1}{2} c_{o}(h)
$$

the Hamiltonian of the system, $\eta_{t=1}=-\partial_{1} \mu\left(o_{t=1}, b\right)$ and (in a local chart)

$$
\left\{\begin{aligned}
\frac{\mathrm{d} o}{\mathrm{~d} t} & =\xi_{o} \circ \zeta_{o}(h) \\
\frac{\mathrm{d} \eta}{\mathrm{d} t} & =-\frac{\partial \mathcal{H}}{\partial o} \\
\frac{\partial \mathcal{H}}{\partial h} & =0
\end{aligned}\right.
$$

Proof. In this proof we suppose that $\mathcal{O}$ is an open subset of $\mathbb{R}^{N}$. As previously we associate to each $h \in L^{2}([0,1], H)$ the trajectory $o^{h}$ of $\mathcal{O}$ such that $o_{t=0}=a$ and $\dot{o}_{t}^{h}=\xi_{o_{t}} \circ \zeta_{o_{t}}\left(h_{t}\right)$. For each $h \in L^{2}([0,1], H), o^{h}$ is absolutely continuous on $[0,1]$ (see Proposition 2) and then belongs to $H_{a}^{1}([0,1], \mathcal{O})$ (elements in $H^{1}([0,1], \mathcal{O})$ starting at $a)$. We define the new functional $\tilde{J}:(o, h) \in H^{1}([0,1], \mathcal{O}) \times L^{2}([0,1], H) \mapsto$ $\int_{0}^{1} c_{o_{t}}\left(h_{t}\right) \mathrm{d} t+\mu\left(o_{t=1}, b\right)$. Then $h \in L^{2}([0,1], H)$ minimizes $J$ if and only if $\left(o^{h}, h\right)$ minimizes $\tilde{J}$ under the constraint $0=\Gamma(o, h) \doteq \dot{o}-\xi_{o} \circ \zeta_{o}(h)$. Functions $\tilde{J}$ and $\Gamma$ are of class $C^{1}$ and $\partial_{o} \Gamma$ is an is an isomorphism for each $o \in H^{1}([0,1], \mathcal{O})$. Indeed let $o \in H^{1}([0,1], \mathcal{O})$ and $\alpha \in L^{2}\left([0,1], \mathbb{R}^{N}\right)$, we can define $\delta o \in H_{0}^{1}\left([0,1], \mathbb{R}^{N}\right)$ by $\delta o(t=0)=0$ and $\dot{\delta o}=\partial_{o}\left(\xi_{o} \circ \zeta_{o}(h)\right)+\alpha$ (the solution is well defined). Then $\alpha=\partial_{o} \Gamma(o, h) \cdot \delta o$ and therefore $\partial_{o} \Gamma(o, h)$ is surjective. Moreover $\partial_{o} \Gamma(o, h)$ is injective as for $\delta o \in H_{0}^{1}\left([0,1], \mathbb{R}^{N}\right), \partial_{o} \Gamma(o, h) \delta o=0$ implies $\delta o=0$ by Cauchy uniqueness. We therefore deduce (using the implicit function theorem) that $\Gamma^{-1}(\{0\})$ is a manifold. Let $(o, h)$ be a minimizer of $\widetilde{J}$ over the set $\Gamma^{-1}(\{0\})$. From [28] (Theorem 4.1) it can be shown that there exists a non trivial Lagrange multiplier $\eta \in L^{2}\left(0,1, \mathbb{R}^{N}\right)^{\star}=$ $L^{2}\left(0,1, \mathbb{R}^{N, \star}\right)$ such that $\mathrm{d} \tilde{J}_{(o, h)}+\left(d \Gamma_{(o, h)}\right)^{\star}(\eta)=0$. It is shown in [6] that $\dot{\eta}=-\partial_{o} \mathcal{H}$ and $\partial_{h} \mathcal{H}=0$.

Remark 9. As $c_{o}$ is positive definite, there exists an invertible symmetric operator $C: o \in \mathcal{O} \mapsto C_{o} \in L\left(H, H^{\star}\right)$ such that for all $(o, h) \in \mathcal{O} \times H, c_{o}(h)=\left(C_{o} h \mid h\right)$. Then 
the third equality in (3.1) allows to compute $h: h=C_{o}^{-1} \rho_{o}^{\star} \eta$ with $\rho_{o}=\xi_{o} \circ \zeta_{o}$ and $\rho_{o}^{\star}$ such that $\left(\eta \mid \rho_{o}(h)\right)_{T_{o} \mathcal{O}}=\left(\rho_{o}^{\star} \eta \mid h\right)_{H}$.

We define the reduced Hamiltonian

$$
\mathcal{H}_{r}(o, \eta) \doteq \mathcal{H}\left(o, \eta, C_{o}^{-1} \rho_{o}^{\star}(\eta)\right)=\frac{1}{2}\left(\rho_{o}^{\star}(\eta) \mid C_{o}^{-1} \rho_{o}^{\star}(\eta)\right)=\frac{1}{2} c_{o}\left(C_{o}^{-1} \rho_{o}^{\star}(\eta)\right)
$$

and as $\nabla_{h} \mathcal{H}=0$, the system of equations (3.1) can be written:

$$
\left\{\begin{array}{l}
\frac{\mathrm{d} o}{\mathrm{~d} t}=\frac{\partial \mathcal{H}_{r}}{\partial \eta}=\rho_{o}\left(C_{o}^{-1} \rho_{o}^{\star}(\eta)\right) \\
\frac{\mathrm{d} \eta}{\mathrm{d} t}=-\frac{\partial \mathcal{H}_{r}}{\partial o}
\end{array}\right.
$$

Proposition 7. If the module $M$ is $C^{j}$ of order $\ell$ with $j, \ell \geq 2$, then solutions of Equation (3.2) exist for any $\left(o_{t=0}, \eta_{t=0}\right)$ and are totally defined by these initial values.

Proof. In this case, $(o, \eta) \mapsto H_{r}(o, \eta)$ is of class at least $C^{2}$ so $(o, \eta) \mapsto\left(\frac{\partial \mathcal{H}_{r}}{\partial \eta},-\frac{\partial \mathcal{H}_{r}}{\partial o}\right)$ is at least $C^{1}$.

Thus, by choosing an initial momentum $\eta \in T_{o}^{*} \mathcal{O}$ one can generate an optimal trajectory starting at $a$. Optimal trajectories are parametrized by initial values of the geometrical descriptor and the momentum, so in dimension $2 \times\left(\operatorname{dim}\left(\mathcal{O}^{1}\right)+\operatorname{dim}(\mathcal{F})\right)$. Remark 10. It is important to note that even though we have $h=\left(h^{1}, 0\right), \rho_{o}^{\star}(\eta) \neq$ $\rho_{o^{1}}^{1, \star}\left(\eta^{1}\right)$ (with $\eta=\left(\eta_{1}, \eta^{2}\right) \in T_{o^{1}}^{*} \mathcal{O}^{1} \times T_{f}^{*} \mathcal{F}$ and $\left.\rho_{o^{1}}^{1}=\xi_{o^{1}}^{1} \circ \zeta_{o^{1}}^{1}\right)$. Then, even though the component of geometrical descriptor belonging to $\mathcal{F}$ generates only a null vector field, its initial momentum has an influence on the trajectory.

Remark 11. From Equation (3.1) we re-deduce that cost the $c_{o}(h)$ is constant along minimizing trajectories. Indeed let $(o, h)$ be such an optimal trajectory and let $\eta$ be the trajectory of momenta, such that $(o, \eta, h)$ satisfies Equation (3.1). Then, as $\rho_{o}(h)=\xi_{o} \circ \zeta_{o}(h)=\nabla_{\eta} \mathcal{H}$, one gets: $\frac{\mathrm{d} \mathcal{H}}{\mathrm{d} t}=\partial_{o} \mathcal{H} \frac{\mathrm{d} o}{\mathrm{~d} t}+\partial_{\eta} \mathcal{H} \frac{\mathrm{d} \eta}{\mathrm{d} t}+\partial_{h} \mathcal{H} \frac{\mathrm{d} h}{\mathrm{~d} t}=\left(\nabla_{o} \mathcal{H}, \nabla_{\eta} \mathcal{H}\right)-$ $\left(\nabla_{\eta} \mathcal{H}, \nabla_{o} \mathcal{H}\right)=0$. So the Hamiltonian is constant along optimal trajectories and as it is equal to half of the cost, $\int_{0}^{1} c_{o_{t}}\left(h_{t}\right) \mathrm{d} t=c_{o_{t=0}}\left(h_{t=0}\right)$ along minimizing trajectories.

4. Modular analysis of shape variability. The goal of this section is to explain how the previous geometrical construction may be used to infer statistical properties from a series of $P$ shape data $f_{\text {target }}^{k}$ belonging to a common shape space $\mathcal{F}$ of order $\ell \geq 2$. In order to do so we will use a derivation of the well-known notion of atlas which corresponds in the literature [13, 19, 46] to one shape, named template and corresponding to a mean shape, associated for each shape data with a deformation transporting the template as close a possible to the shape data. In our context we set a deformation module $\tilde{M}=(\tilde{\mathcal{O}}, \tilde{H}, \tilde{\zeta}, \tilde{\xi}, \tilde{c})$ (satisfying the UEC) and we will consider large deformations that can be generated by this deformation module. As in Section 3.2.2 we build $M=(\mathcal{O}, H, \zeta, \xi, c)$ as the compound module of $\tilde{M}$ and the silent deformation module induced from $\mathcal{F}$. The estimation of the atlas may be done in a coherent Bayesian framework. However, the derivation of a Bayesian approach as in [3] is out of the scope of this paper, and we propose here a more straightforward extension of the geometrical construction of Section 3.2.2. It is the analogue of the concept of Fréchet mean, which has been used intensively in the field of Computational Anatomy [29, 24, 14]. Computing an atlas of the data set will then correspond to computing one initial value of the geometrical descriptor, and modular large deformations generated by it, bringing its silent component as close as possible to the target shapes. 
Geometrical descriptors of the silent deformation module will be referred to as the template shape and play the role of an "average" of the data set. In the spirit of [14], the user provides an example of template shape with the desired topology (i.e. number of points, edges between points, number of connected components, etc..), and the atlas construction method will optimize its shape (i.e. the position of the points or vertices) to be at the "center" of the training samples. The active deformation module is a dictionary, in which the shape variations seen in the data may be decomposed. The choice of the types of modules is left to the user, whereas the initial geometrical descriptors are to be optimized for a given training shape data set.

The method needs to find at the same time the $P$ modular large deformations $\phi^{k}$, so that each warped template resembles as much as possible one of the target shapes $f_{\text {target }}^{k}$. Given the initial position of geometrical descriptors, modular large deformations that need to be estimated are parametrized by initial momenta. So finally the method will estimate one initial position of geometrical descriptors and $P$ initial momenta. Then computing the atlas of shapes $f_{\text {target }}^{k}, k=1 \cdots P$ with the deformation module $\tilde{M}$ amounts to minimizing:

$$
E\left(o_{\text {temp }},\left(\eta_{0}^{k}\right)_{k}\right)=\sum_{k=1}^{P} c_{\text {otemp }}\left(h^{o_{\text {temp }}, \eta_{0}^{k}}\right)+\frac{1}{\lambda^{2}} \mu\left(\phi^{o^{k}, \eta^{k}} \cdot f_{\text {temp }}, f_{\text {target }}^{k}\right),
$$

where for each $k,\left(o^{k}, \eta^{k}\right)$ starts at $\left(o_{t e m p}, \eta_{0}^{k}\right)$ and satisfies, with $\mathcal{H}:(o, \eta, h) \in$ $T^{*} \mathcal{O} \times H \mapsto\left(\eta \mid \xi_{o}\left(\zeta_{o}(h)\right)\right)-\frac{1}{2} c_{o}(h)$,

$$
\left\{\begin{aligned}
h^{k} & =h^{o^{k}, \eta^{k}} \doteq C_{o^{k}}^{-1} \rho_{o^{k}}^{\star} \eta^{k} \\
\frac{\mathrm{d} o^{k}}{\mathrm{~d} t} & =\xi_{o^{k}}\left(\zeta_{o^{k}}\left(h^{k}\right)\right. \\
\frac{\mathrm{d} \eta^{k}}{\mathrm{~d} t} & =-\frac{\partial \mathcal{H}}{\partial o}\left(o^{k}, \eta^{k}, h^{k}\right),
\end{aligned}\right.
$$

and $\phi^{o^{k}}, h^{k}=\varphi_{t=1}^{v^{k}}$ with $v^{k}=\zeta_{o^{k}}\left(h^{k}\right)$. The first term of this sum will be referred to as the Regularity term while the second one will be called the Data term. The parameter $\lambda$ is set. In practice it is empirically chosen so that the regularity and data terms are of the same order of magnitude when the energy $E$ is close to its minimum value.

Note that the initial value of geometrical descriptor $o_{\text {temp }}$ is common to all subjects but that the trajectory $o^{k}$ (starting at $o_{\text {temp }}$ ) obtained by integrating Equation (4.2) is specific to each subject as it depends on the initial momentum $\eta_{0}^{k}$, which is specific to each subject.

We emphasize here that the estimated $o_{\text {temp }}=\left(o_{\text {temp }}^{1}, f_{\text {temp }}\right)$ has two components. The second one $f_{\text {temp }}$ is the template and corresponds to an average of the data set, while the first one $o_{\text {temp }}^{1}$ is a common geometric characterization of the variability among the population of shapes.

5. Numerical experiments. We present here two simple examples to illustrate how our generic framework can be used. The first one illustrates how a prior can be incorporated in the study of a population through the choice of the deformation module. The second one illustrates the fact that our framework can be used when there is no strong prior on the population under study.

We do not investigate here the problem of the module's choice: we suppose that the deformation module to use is given. The stability with respect to the parameters of the modules (such as the scales of the deformations) is also out of the scope of this article. However we tested different values of these parameters and we obtained 

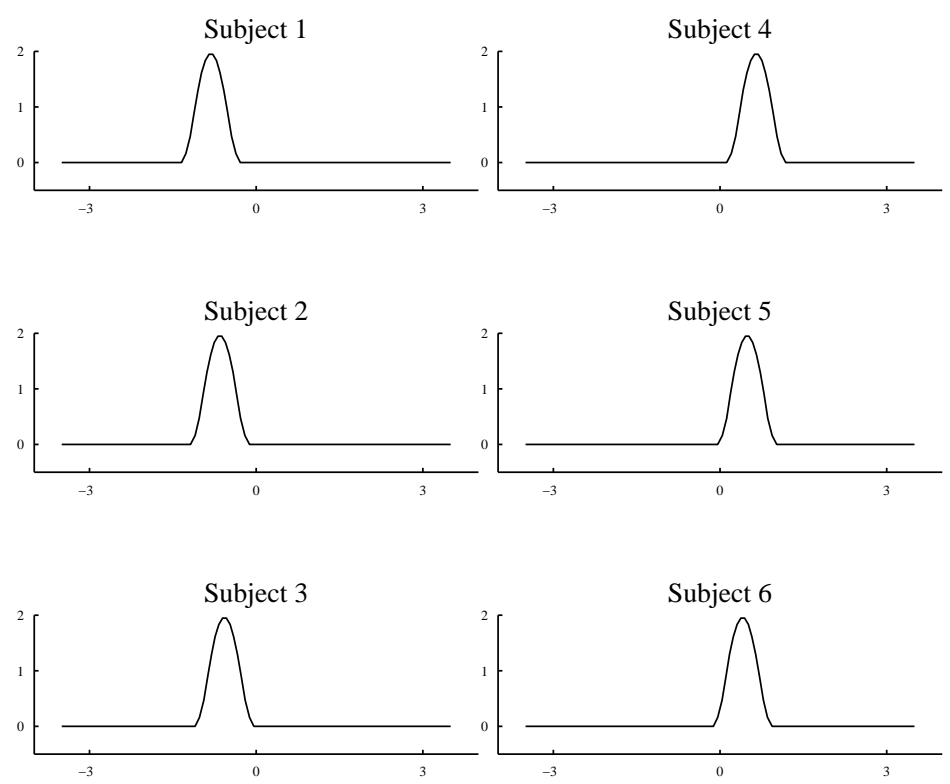

FIG. 5.1. Target shapes.
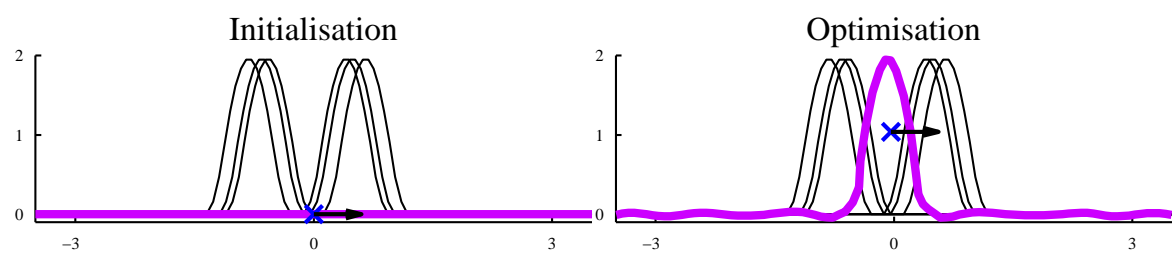

Fig. 5.2. Prior: horizontal displacement. Template at time $t=0$ (purple curve) and other geometrical descriptor (blue cross). Targets in black. The black vector is the fixed parameter that defines the direction of the translation. Left: before optimisation. Right: after optimisation.

similar results (which are not presented here, a deep discussion will be the object of another article). The choice of the deformation module can be critical in practice, it corresponds to a choice of vocabulary and learning the most adapted vocabulary for given data might be done following a Bayesian strategy.

5.1. Building an atlas using a prior. In this section, we study the set of shapes presented in Figure 5.1 : each shape is a one-dimensional curve with a hump in a two-dimensional space. They are represented using the framework of varifolds [12], so without canonical parametrization or points of correspondence between them. Three of them have the hump rather on their left part, and three others have it on their right part, all at variable locations. Intuitively, there are two possible descriptions of the variability of this collection of shapes. One possibility is to consider that shapes derive from a "template" shape with one central hump by random translations of the hump in either direction. Another possibility is to consider that shapes derive from "template" shape by unfolding the hump in one place and fold a hump at another place. These two models of shape variability would explain the observed samples 

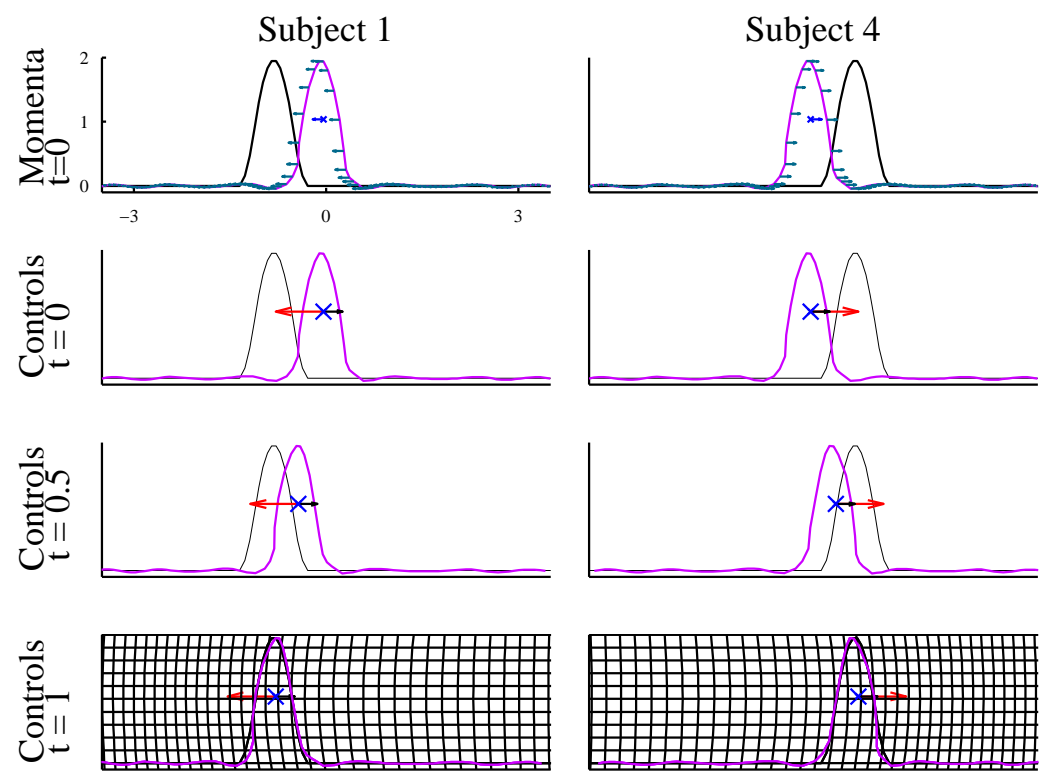

FIG. 5.3. Prior: horizontal displacement. Template at time $t$ (in purple), target shapes are in black. Other geometrical descriptor at time $t$ (blue cross). First line: momenta at $t=0$ (in marine blue are momenta attached to the template's points and in blue the one attached to center of the translation with fixed direction). Three bottom lines : the black vector is the fixed parameter that defines the direction of the translation, controls at $t$ are represented by the length of the red arrow.

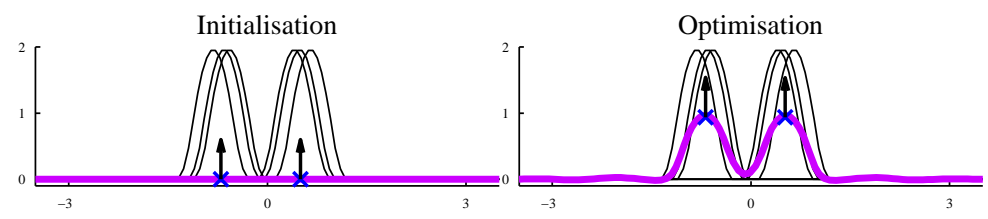

FIG. 5.4. Prior: vertical displacements. Template at time $t=0$ (purple curve) and other geometrical descriptor (blue crosses). The black vector is the fixed parameter that defines the direction of the translation. Targets in black. Left: before optimisation. Right: after optimisation.

equally well, and the problem is undecidable without assuming priors on the solution. Determining the template reflecting the structure in the data set, and allowing to study its variability is a well-known problem in computer vision and shape analysis, as described in $[27,32]$ for instance. If no point-correspondence is assumed, the current implementation of most statistical shape analysis techniques will give one or the other solution in a complex way depending mostly on implementation choices, initialisation, regularisation techniques, etc.

We illustrate here that our approach based on modular deformations allows the user to decide beforehand which solution should be favored. The addition of such a prior on the sought solution is possible by the design of relevant modules.

To obtain a description of shape variability based on horizontal translations of the hump, we use a user-defined deformation module, which generates a vector field that is always a horizontal translation (see Section 2.3.5, here we use a constant direction 

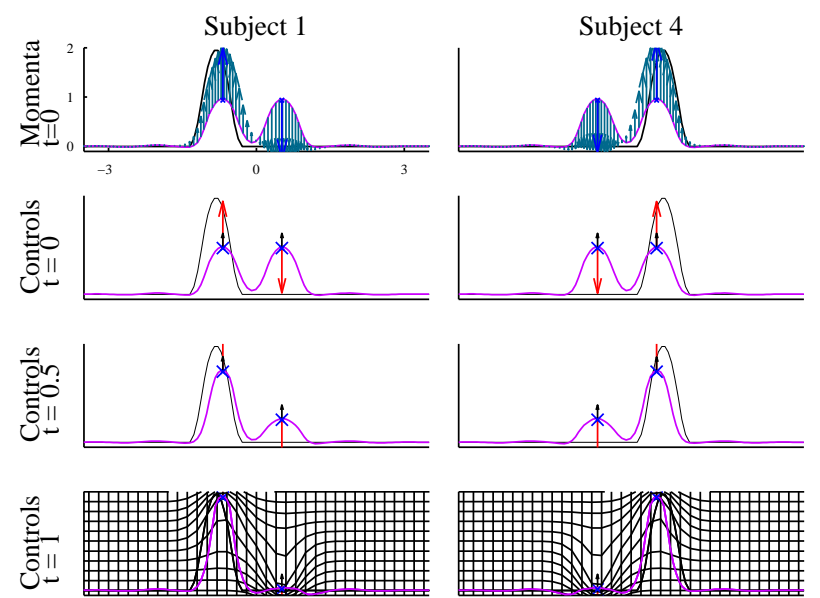

FIG. 5.5. Prior: vertical displacements. Template at time $t$ (in purple), target shapes are in black. Other geometrical descriptor at time $t$ (blue crosses). First line : momenta at $t=0$ (in marine blue are momenta attached to the template's points and in blue the ones attached to centers of translations with fixed direction). Three bottom lines : black vectors are the fixed parameter that defines the directions of translations, controls at $t$ are represented by the lengths of red arrows.

of translation) at a large scale $\sigma=3 \mathrm{~cm}$ (where the height of the hump is $2 \mathrm{~cm}$ ). We initialize the template curve with a shape with no hump, include it into a silent module and combine it with the translation module. We minimize the Functional (4.1), where $\mu$ is the varifold distance between the curves, which measures how well two curves are superimposed without the need to have point-correspondence and consistent orientation [12]. The result of the minimisation is the optimal geometrical descriptors of the compound module, here the position of the vertices of the template curve and the base point of the translation, and one initial momentum per target shape characterising the deformation of the template curve to the corresponding target shape. In Figure 5.2 (left part) we present the initialisation of the template and the geometrical descriptor, momenta $\eta_{0}^{k}$ are initialized at zero. The fixed horizontal vector $u=(1,0)$ is plotted in black, it is not optimized during the gradient descent and does not evolve during the integration of the trajectory of the vector field. In Figure 5.2 (right part) we display the optimized shared parameters of optimal trajectories: the optimized template and position for the geometrical descriptor at time $t=0$. The diffeomorphic deformation from the template to one target is parametrized by values at time $t=0$ of the template, the geometrical descriptor and the momentum (dual variable of the geometrical descriptor). We present in Figure 5.3, for two subjects, this parametrization of trajectories (first row), and also the transport of the template to targets with the geometrical descriptor and the control (last three rows). As the control is scalar, it is plotted as the length of the vector of the translation: the unit horizontal vector (fixed parameter) is plotted in black and in red is plotted this vector multiplied by the scalar control.

By contrast, we may decide to describe the variability in this same data set by using folding/unfolding pattern. The corresponding prior in the deformation model is encoded by one deformation module generating vector fields that are always a sum of two vertical translations at a fixed small scale $\sigma$ (see Section 2.3.5, here we use $\sigma=0.4$ 
and constant directions of translations). In Figure 5.4 can be seen the template and other geometrical descriptors before and after optimisation, as well as fixed vertical vectors . Parametrisation of minimizing trajectories, and trajectories of the template, geometrical descriptors and controls are plotted in Figure 5.5 for two subjects. As previously, controls can be seen in the lengths of vertical vectors, black vectors being fixed vertical unit vectors.

These two experiments show that one may obtain different templates and deformations from the same data set by using different prior on shape variability. Our method allows the user to easily encode such prior in an intuitive and controlled manner by the design of suitable modules.

5.2. An example of atlas with a weak prior. We present now an example where there is no obvious deformation module, which can be the case in practice. We consider here the five shapes of skulls presented in 5.6. Similarly to previous examples, shapes are encoded as varifolds [12] so that no point-correspondence is assumed. As we do not have a strong prior on the deformations we want to use to study this population, we combine several deformation modules which generate deformations that can be easily interpreted. They correspond to local deformations at different scales which are chosen following a simple process from the data: as the sizes of the skulls are approximately 200, we chose three different scales: 200, 100 and 50. The initial geometrical descriptors are initialized on regular grids whose step sizes depend on the scale. More precisely we use a combination of 7 deformation modules generating respectively one translation at scale $\sigma=200$ (see Section 2.3.3), one rotation at scale $\sigma=200$ (see Section 2.3.1), one sum of two rotations at scale $\sigma=100$, one sum of two scalings at scale $\sigma=100$ (see Section 2.3.1), one sum of four rotations at scale $\sigma=50$, one sum of four scalings at scale $\sigma=50$ and one sum of 16 translations of directions updated by adjoint action at scale $\sigma=50$ (see Section 2.3.5).

The first two deformation modules (those at large scale) enable to perform rigid registration simultaneously with finer deformations. At the smallest scales, translations with transported direction have shared initial positions and directions for all subjects, and their directions are transported by the flow. Using this deformation module instead of one generating unconstrained translations as defined in Section 2.3.3, allows to interpret the initial vector of these translations as directions of greatest variability among the population (at the small scale).

The initial distribution of the geometrical descriptors on a regular lattice and the template before optimisation can be seen in Fig. 5.7. Geometrical descriptors are updated during optimisation to regions of interest. Parametrization of minimizing trajectories (template, initial other geometrical descriptors and momenta) and trajectories of template, geometrical descriptors and controls can be seen in Figure 5.8 for three skulls.

From these results one can see, for example, that the size of the cranium is a feature that varies importantly amongst the population as one center of the two local scalings at scale 100 has moved to this area during optimisation, and trajectories of the corresponding control are different for all skulls: negative for subject 1 (the red triangle is very small so the control is highly negative: his cranium is smaller than the template), close to zero for subject 3 (the red triangle is almost of the size of the blue one: his cranium is almost of same size as the template) and positive for subject 5 (the red triangle is bigger than the blue one: his cranium is bigger than the template). Other features of great variabilities can be deduced from this results such as, for instance, the shape of the back of the head by inspecting the initial value of 


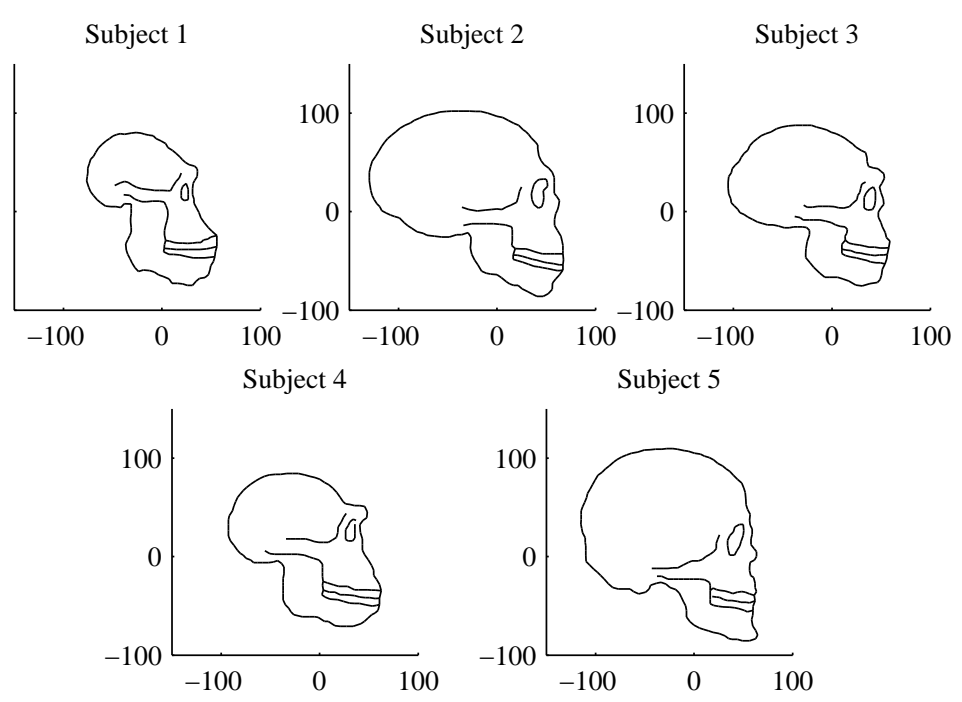

FIG. 5.6. Target shapes.
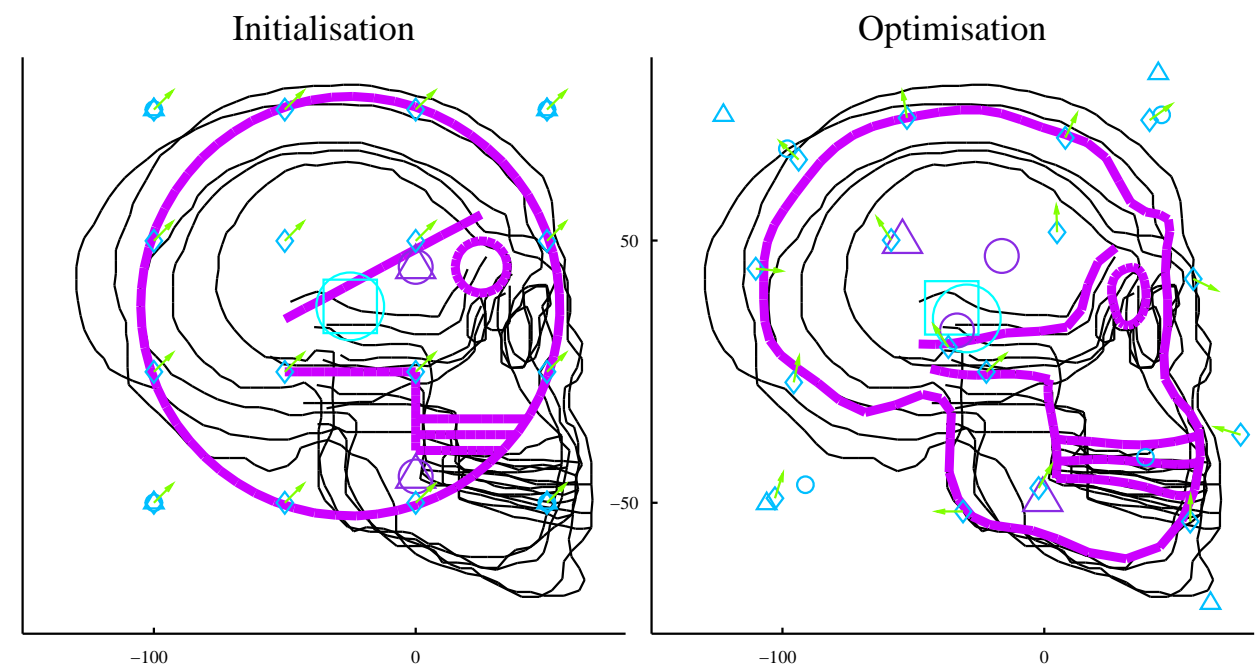

FIG. 5.7. Template at time $t=0$ (purple shape) and geometrical descriptors: $\square$ (translations), $\triangle$ (rotations), $\triangle$ (scalings) and green vectors attached to $\diamond$ (translations with updated directions). The color and size of markers represent the scale $(200,100,50)$. Black shapes are the targets. Left: before optimisation. Right: after optimisation.

vector of the corresponding translation with updated direction.

This experiment illustrates in a more realistic case how the variability seen in a shape data set may be decomposed into a series of local non-linear and multiscale deformation patterns. 


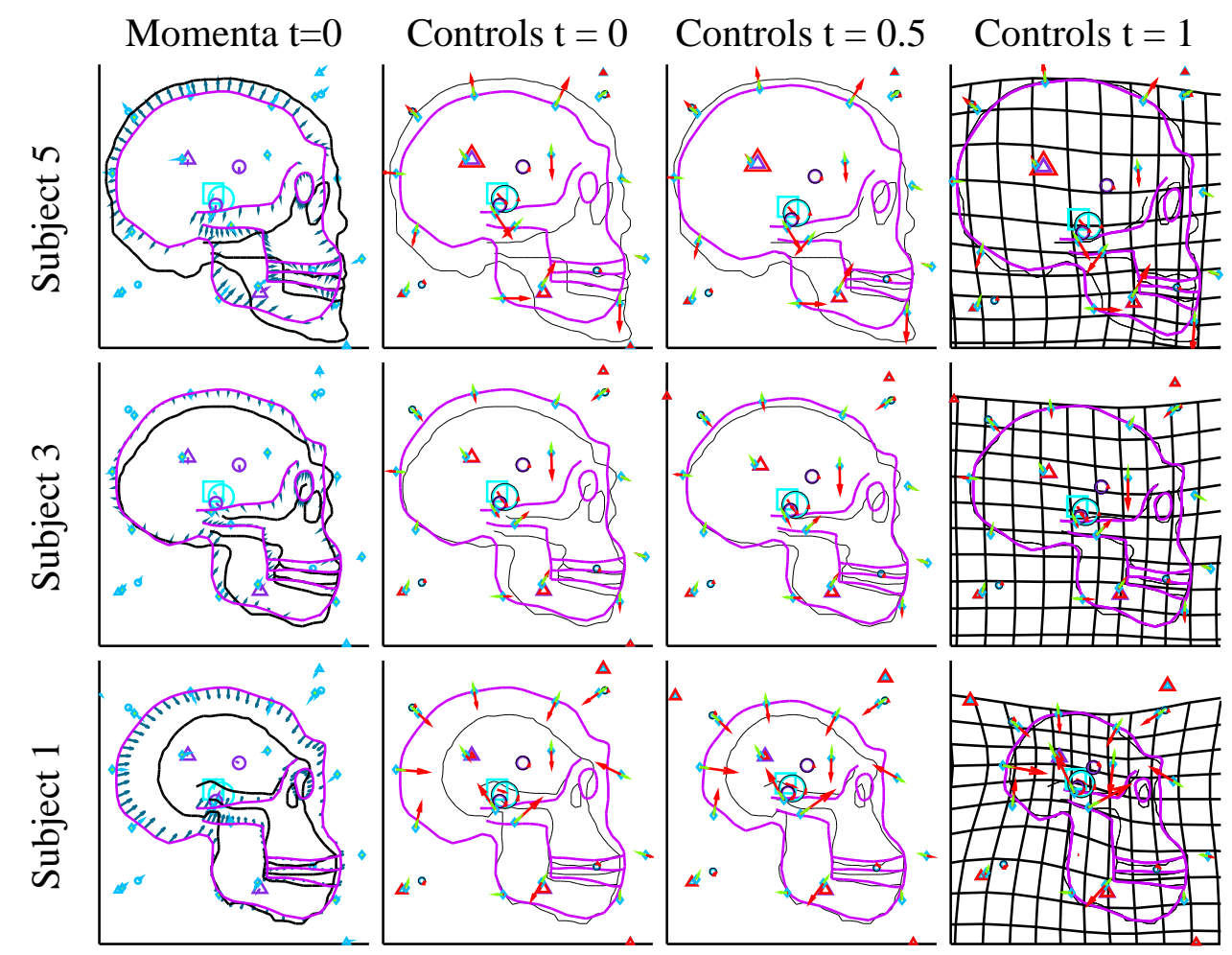

FIG. 5.8. Template at time $t$ (in purple), target shapes are in black. Geometrical descriptors at time $t: \square$ (translations), $\circ$ (rotations), $\triangle$ (scalings) and green vectors attached to $\diamond$ (translations with updated directions). The color and size of markers represent the scale $(200,100,50)$. Left column: Momenta at $t=0$. Three right-hand columns: Controls at time $t$ in red (vector for the translation, portion of the blue circle coloured in red for rotations, ratio between the blue and red triangles for scalings and length of the red vectors for translations with updated direction).

6. Conclusion. In this paper we defined a coherent mathematical framework to build locally constrained diffeomorphic deformations via the introduction of a new concept: deformation modules. The modules constrain the driving velocity field of the deformation to belong to particular subspaces of admissible vector fields. The flow of such sub-Riemannian trajectories of vector fields forms a modular large deformation. These deformations act both on the shapes (namely points, meshes or images) that are embedded into the ambient space, and on the geometrical parameters of the deformation modules themselves, so that they are all transported during the integration of the flow equations. We consider the shapes to be deformed as geometrical parameter of a particular module, called "silent" module, so that shapes and active geometrical descriptors alike belong to a common "shape space". We show that our construction enables the definition of a sub-Riemannian distance on this shape space, and that normal geodesics are parametrized in finite dimension by the initial momentum.

We presented how these deformations may be used to summarize the variability observed in a series of data shapes. The proposed method estimates a "template shape", as a Fréchet mean, and decomposes the observed variability into a dictionary of deformation modules encoding specific local deformation patterns. The relative position of each example shape with respect to the template shape on the sub-Riemannian 
manifold is encoded into the initial momentum. These descriptors of shape variability may be then used for statistical purposes, such as classification, clustering, or regression against covariates.

It is worth noting that the dimension of the initial momentum is larger than the number of control parameters in the modules, since it is the sum of the dimension of geometrical descriptors of the active modules and the number of points in the shapes to be deformed, namely the geometrical descriptor of the silent module. Therefore, the variability is usually encoded in much higher dimension than the number of degrees of freedom of the deformations. There is an interplay between the momentum attached to the active modules and the one attached to the shapes, which enriches the possible dynamics of control parameters in the active modules. In particular, the initial value of the control parameters does not uniquely determine the subsequent deformation, neither do initial momenta of active deformation modules alone. Nonetheless, it is not clear what the optimal number of parameters to describe the variability of a given data set is. One may want to automatically learn this number using sparse penalties in the spirit of [15]. It is also an open question to know whether the same deformations may be obtained in a purely Riemannian framework, where the dimension of the initial momentum exactly matches the number of control parameters, that is the number of degrees of freedom of the deformations.

We illustrated our method with two examples. The first one illustrates an important advantage of our framework : incorporating a prior in the deformation model. In particular we presented how two different deformation modules, corresponding to two different priors, lead to two different template shapes. These two templates both correspond to a mean of the data shapes, but they are built with different vocabularies to study differences between shapes. A very interesting, and largely open, question would be to address how to select the deformation module that would be the most adapted to a given population. 
Appendix A. Summary of notation. We summarize here the notation we use:

- $M=(\mathcal{O}, H, \zeta, \xi, c)$ is the deformation module $M$ with space of geometrical descriptors $\mathcal{O}$, space of controls $H$, infinitesimal action $\xi$, field generator $\zeta$ and cost $c$.

- $o$ refers in general to geometrical descriptors.

- $h$ refers in general to controls.

- $v$ refers in general to vector fields.

- $V$ refers in general to the space of vector fields thanks to which a module satisfies UEC.

- $\varphi$ refers in general to a path of diffeomorphisms.

- $\varphi^{v}$ : flow of $v$.

- $\phi$ refers in general to a diffeomorphism.

- $C_{0}^{\ell}\left(\mathbb{R}^{d}\right)$ : space of vector fields of class $C^{\ell}$ on $\mathbb{R}^{d}$ whose derivatives of order less than or equal to $\ell$ converge to zero at infinity.

- Diff $0_{0}\left(\mathbb{R}^{d}\right)$ : space of $C^{\ell}$-diffeomorphisms of $\mathbb{R}^{d}$ that converge to identity at infinity.

- $K_{\sigma}$ : scalar kernel of the scalar Gaussian RKHS of scale $\sigma>0$.

- $X^{*}$ : dual of a vector space $X$.

- $(\delta \mid x)_{X^{*}}$ : application of $\delta \in X^{*}$ to $x \in X$ (for $X$ a vector space).

- $\langle\cdot, \cdot\rangle_{X}$ : inner product of vector space $X$.

Appendix B. Summary of the used deformation modules. We summarize here the deformation modules (in order of appearance) which are used in the numerical experiments of Section 5 , they are all in dimension 2.

\begin{tabular}{|c|c|c|c|}
\hline Deformation module & Geometrical descriptor & Control & Generated vector Field \\
\hline $\begin{array}{c}\text { Sum of } D \text { local } \\
\text { translations at scale } \sigma \\
\text { with a fixed direction } u\end{array}$ & $\begin{array}{c}\text { set of } D \text { points } \\
o=\left(z_{j}\right)_{1 \leq j \leq D}\end{array}$ & $\begin{array}{l}\text { set of } D \text { scalars } \\
h=\left(\alpha_{j}\right)_{1 \leq j \leq D}\end{array}$ & $\zeta_{o}(h)=\sum_{j=1}^{D} \alpha_{j} K_{\sigma}\left(z_{j}, \cdot\right) u$ \\
\hline $\begin{array}{c}\text { Sum of } D \text { local rotations } \\
\text { at scale } \sigma\end{array}$ & $\begin{array}{l}\text { set of } D \text { points } \\
o=\left(x_{j}\right)_{1 \leq j \leq D}\end{array}$ & $\begin{array}{l}\text { set of } D \text { scalars } \\
h=\left(\alpha_{j}\right)_{1 \leq j \leq D}\end{array}$ & $\begin{array}{c}\zeta_{o}(h)=\sum_{\substack{1 \leq j \leq D \\
1 \leq k \leq 3}} \alpha_{j} K_{\sigma}\left(z_{k}\left(x_{j}, \sigma\right), \cdot\right) d_{k} \\
\text { with } d_{1}=\left(\frac{1}{2}, \frac{1}{2} \sqrt{3}\right), d_{2}=\left(\frac{1}{2},-\frac{1}{2} \sqrt{3}\right), \\
d_{3}=(-1,0), z_{1}\left(x_{j}, \sigma\right)=x_{j}+\frac{\sigma}{3}\left(\frac{1}{2} \sqrt{3},-\frac{1}{2}\right), \\
z_{2}\left(x_{j}, \sigma\right)=x_{j}+\frac{\sigma}{3}\left(-\frac{1}{2} \sqrt{3},-\frac{1}{2}\right), \\
z_{3}\left(x_{j}, \sigma\right)=x_{j}+\frac{\sigma}{3}(0,1)\end{array}$ \\
\hline Local translation & one point $O$ & one vector $h$ & $\zeta_{o}(h)=K_{\sigma}(o, \cdot) h$ \\
\hline $\begin{array}{c}\text { Sum of } D \text { local } \\
\text { translations at scale } \sigma \\
\text { with a direction updated } \\
\text { by adjoint action }\end{array}$ & $\begin{array}{l}\text { set of } D \text { points } \\
\text { and } D \text { vectors } \\
o=\left(z_{j}, u_{j}\right)_{1 \leq j \leq D}\end{array}$ & $\begin{array}{l}\text { set of } D \text { scalars } \\
h=\left(\alpha_{j}\right)_{1 \leq j \leq D}\end{array}$ & $\zeta_{o}(h)=\sum_{j=1}^{D} \alpha_{j} K_{\sigma}\left(z_{j}, \cdot\right) u_{j}$ \\
\hline $\begin{array}{c}\text { Sum of } D \text { local scalings } \\
\text { at scale } \sigma\end{array}$ & $\begin{array}{l}\text { set of } D \text { points } \\
o=\left(x_{j}\right)_{1 \leq j \leq D}\end{array}$ & $\begin{array}{l}\text { set of } D \text { scalars } \\
h=\left(\alpha_{j}\right)_{1 \leq j \leq D}\end{array}$ & $\begin{array}{c}\zeta_{o}(h)=\sum_{\substack{1 \leq j \leq D \\
1 \leq k \leq 3}} \alpha_{j} K_{\sigma}\left(z_{k}\left(x_{j}, \sigma\right), \cdot\right) d_{k} \\
\text { with } d_{1}=\left(\frac{1}{2} \sqrt{3},-\frac{1}{2}\right), d_{2}=\left(-\frac{1}{2} \sqrt{3},-\frac{1}{2}\right), \\
\quad d_{3}=(0,1), z_{k}\left(x_{j}, \sigma\right)=x_{j}+\frac{\sigma}{3} d_{k}\end{array}$ \\
\hline
\end{tabular}




\section{REFERENCES}

[1] A. Agrachev, D. Barilari, and U. Boscain. Introduction to Riemannian and Sub-Riemannian geometry. 2014

[2] S. Allassonnière, Y. Amit, and A. Trouvé. Towards a coherent statistical framework for dense deformable template estimation. Journal of the Royal Statistical Society: Series B (Statistical Methodology), 69(1):3-29, 2007.

[3] S. Allassonnière, E. Kuhn, A. Trouvé, et al. Construction of bayesian deformable models via a stochastic approximation algorithm: a convergence study. Bernoulli, 16(3):641-678, 2010.

[4] S. Arguillere. Géométrie sous-riemannienne en dimension infinie et applications à l'analyse mathématique des formes. PhD thesis, Paris 6, 2014.

[5] S. Arguillere. The general setting for shape analysis. arXiv:1504.01767, 2015.

[6] S. Arguillere, E. Trélat, A. Trouvé, and L. Younes. Shape deformation analysis from the optimal control viewpoint. Journal de mathématiques pures et appliquées, 2015.

[7] V. Arsigny, O. Commowick, N. Ayache, and X. Pennec. A fast and log-euclidean polyaffine framework for locally linear registration. Journal of Mathematical Imaging and Vision, $33(2): 222-238,2009$.

[8] V. Arsigny, X. Pennec, and N. Ayache. Polyrigid and polyaffine transformations: a novel geometrical tool to deal with non-rigid deformations-application to the registration of histological slices. Medical image analysis, 9(6):507-523, 2005.

[9] B. B. Avants, C. L. Epstein, M. Grossman, and J. C. Gee. Symmetric diffeomorphic image registration with cross-correlation: evaluating automated labeling of elderly and neurodegenerative brain. Medical image analysis, 12(1):26-41, 2008.

[10] M. Bauer, M. Bruveris, and P. W. Michor. Overview of the geometries of shape spaces and diffeomorphism groups. Journal of Mathematical Imaging and Vision, 50(1-2):60-97, 2014.

[11] M. Bruveris, L. Risser, and F.-X. Vialard. Mixture of kernels and iterated semidirect product of diffeomorphisms groups. Multiscale Modeling \&3 Simulation, 10(4):1344-1368, 2012.

[12] N. Charon and A. Trouvé. The varifold representation of non-oriented shapes for diffeomorphic registration. arXiv preprint arXiv:1304.6108, 2013.

[13] G. E. Christensen, R. D. Rabbitt, and M. I. Miller. Deformable templates using large deformation kinematics. Image Processing, IEEE Transactions on, 5(10):1435-1447, 1996.

[14] S. Durrleman, M. Prastawa, N. Charon, J. R. Korenberg, S. Joshi, G. Gerig, and A. Trouvé. Morphometry of anatomical shape complexes with dense deformations and sparse parameters. NeuroImage, 101:35-49, 2014.

[15] S. Durrleman, M. Prastawa, G. Gerig, and S. Joshi. Optimal data-driven sparse parameterization of diffeomorphisms for population analysis. In Information Processing in Medical Imaging, pages 123-134. Springer, 2011.

[16] J. Glaunes. Transport par difféomorphismes de points, de mesures et de courants pour la comparaison de formes et l'anatomie numérique. These de sciences, Université Paris, 13, 2005.

[17] J. Glaunès, M. Vaillant, and M. I. Miller. Landmark matching via large deformation diffeomorphisms on the sphere. Journal of mathematical imaging and vision, 20(1-2):179-200, 2004 .

[18] U. Grenander. Elements of pattern theory. JHU Press, 1996.

[19] U. Grenander and D. M. Keenan. On the shape of plane images. SIAM Journal on Applied Mathematics, 53(4):1072-1094, 1993.

[20] U. Grenander, A. Srivastava, and S. Saini. A pattern-theoretic characterization of biological growth. Medical Imaging, IEEE Transactions on, 26(5):648-659, 2007.

[21] B. Gris. Modular approach on shape spaces, Sub-Riemannian geometry and computational anatomy. PhD thesis, École Normale Supérieure de Cachan, Institut du Cerveau et de la Moelle épinière, 2016.

[22] B. Gris, S. Durrleman, and A. Trouvé. A sub-riemannian modular approach for diffeomorphic deformations. In Geometric Science of Information, pages 39-47. Springer, 2015.

[23] H. Jacobs. Symmetries in lddmm with higher order momentum distributions. arXiv preprint arXiv:1306.3309, 2013.

[24] S. Joshi, B. Davis, M. Jomier, and G. Gerig. Unbiased diffeomorphic atlas construction for computational anatomy. NeuroImage, 23:S151-S160, 2004.

[25] D. G. Kendall. Shape manifolds, procrustean metrics, and complex projective spaces. Bulletin of the London Mathematical Society, 16(2):81-121, 1984.

[26] D. G. Kendall, D. Barden, T. K. Carne, and H. Le. Shape and shape theory, volume 500. John Wiley \& Sons, 2009.

[27] A. C. Kotcheff and C. J. Taylor. Automatic construction of eigenshape models by genetic 
algorithm. In Information Processing in Medical Imaging, pages 1-14. Springer, 1997.

[28] S. Kurcyusz. On the existence and nonexistence of lagrange multipliers in banach spaces. Journal of Optimization Theory and Applications, 20(1):81-110, 1976.

[29] P. Lorenzen, B. C. Davis, and S. Joshi. Unbiased atlas formation via large deformations metric mapping. In Medical Image Computing and Computer-Assisted Intervention-MICCAI 2005, pages 411-418. Springer, 2005.

[30] J. Ma, M. I. Miller, A. Trouvé, and L. Younes. Bayesian template estimation in computational anatomy. NeuroImage, 42(1):252-261, 2008.

[31] K. McLeod, M. Sermesant, P. Beerbaum, and X. Pennec. Spatio-temporal tensor decomposition of a polyaffine motion model for a better analysis of pathological left ventricular dynamics. IEEE transactions on medical imaging, 34(7):1562-1575, 2015.

[32] G. McNeill and S. Vijayakumar. Linear and nonlinear generative probabilistic class models for shape contours. In Proceedings of the 24th international conference on Machine learning, pages 617-624. ACM, 2007.

[33] M. I. Miller, A. Trouvé, and L. Younes. On the metrics and euler-lagrange equations of computational anatomy. Annual review of biomedical engineering, 4(1):375-405, 2002.

[34] M. I. Miller, L. Younes, and A. Trouvé. Diffeomorphometry and geodesic positioning systems for human anatomy. Technology, 2(01):36-43, 2014.

[35] N. Portman. The modelling of biological growth: A pattern theoretic approach. 2010.

[36] L. Risser, F. Vialard, R. Wolz, M. Murgasova, D. D. Holm, and D. Rueckert. Simultaneous multi-scale registration using large deformation diffeomorphic metric mapping. Medical Imaging, IEEE Transactions on, 30(10):1746-1759, 2011.

[37] M.-M. Rohé, N. Duchateau, M. Sermesant, and X. Pennec. Combination of polyaffine transformations and supervised learning for the automatic diagnosis of $\mathrm{lv}$ infarct. In International Workshop on Statistical Atlases and Computational Models of the Heart, pages 190-198. Springer, 2015.

[38] C. Seiler, X. Pennec, and M. Reyes. Capturing the multiscale anatomical shape variability with polyaffine transformation trees. Medical image analysis, 16(7):1371-1384, 2012.

[39] S. Sommer, F. Lauze, M. Nielsen, and X. Pennec. Kernel bundle epdiff: evolution equations for multi-scale diffeomorphic image registration. In Scale Space and Variational Methods in Computer Vision, pages 677-688. Springer, 2011.

[40] S. Sommer, F. Lauze, M. Nielsen, and X. Pennec. Sparse multi-scale diffeomorphic registration: the kernel bundle framework. Journal of mathematical imaging and vision, 46(3):292-308, 2013.

[41] S. Sommer, M. Nielsen, S. Darkner, and X. Pennec. Higher-order momentum distributions and locally affine lddmm registration. SIAM Journal on Imaging Sciences, 6(1):341-367, 2013.

[42] S. Sommer, M. Nielsen, F. Lauze, and X. Pennec. A multi-scale kernel bundle for lddmm: towards sparse deformation description across space and scales. In Information Processing in Medical Imaging, pages 624-635. Springer, 2011.

[43] A. Srivastava, S. Saini, Z. Ding, and U. Grenander. Maximum-likelihood estimation of biological growth variables. In Energy Minimization Methods in Computer Vision and Pattern Recognition, pages 107-118. Springer, 2005.

[44] D. W. Thompson et al. On growth and form. On growth and form., 1942.

[45] A. Trouvé. An infinite dimensional group approach for physics based models in pattern recognition. preprint, 1995.

[46] A. Trouvé. Diffeomorphisms groups and pattern matching in image analysis. International Journal of Computer Vision, 28(3):213-221, 1998.

[47] M. Vaillant, M. I. Miller, L. Younes, and A. Trouvé. Statistics on diffeomorphisms via tangent space representations. NeuroImage, 23:S161-S169, 2004.

[48] T. Vercauteren, X. Pennec, A. Perchant, and N. Ayache. Diffeomorphic demons: Efficient non-parametric image registration. NeuroImage, 45(1):S61-S72, 2009.

[49] L. Younes. Shapes and diffeomorphisms, volume 171. Springer Science \& Business Media, 2010.

[50] L. Younes. Constrained diffeomorphic shape evolution. Foundations of Computational Mathematics, 12(3):295-325, 2012.

[51] L. Younes, F. Arrate, and M. I. Miller. Evolutions equations in computational anatomy. NeuroImage, 45(1):S40-S50, 2009.

[52] W. Zhang, J. A. Noble, and J. M. Brady. Adaptive non-rigid registration of real time 3d ultrasound to cardiovascular $\mathrm{mr}$ images. In Information Processing in Medical Imaging, pages 50-61. Springer, 2007. 